\title{
Turbulent separated shear flow control by surface plasma actuator - Experimental optimization by genetic algorithm approach
}

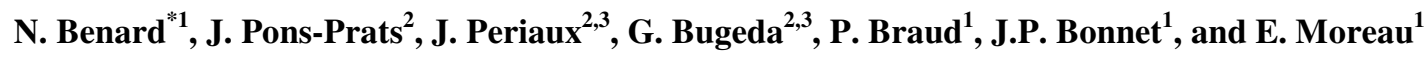 \\ ${ }^{1}$ Institut PPRIME - UPR 3346 - CNRS - Université de Poitiers - ISAE/ENSMA - SP2MI Téléport2 Bd Marie \& Pierre Curie \\ BP 30179, 86962 Futuroscope Chasseneuil Cedex, France \\ ${ }^{2}$ International Center for Numerical Methods in Engineering (CIMNE), c/ Esteve Terrades 508860 Castelldefels, Spain. \\ ${ }^{3}$ Universitat Politècnica de Catalunya (UPC), c/ Gran Capità s/n 08034 Barcelona, Spain
}

\begin{abstract}
The potential benefits of active flow control are no more debated. Among many others applications, flow control provides an effective mean for manipulating turbulent separated flows. Here, a non-thermal surface plasma discharge (dielectric barrier discharge) is installed at the step corner of a backward-facing step $\left(U_{0}=15 \mathrm{~m} / \mathrm{s}\right.$, $\mathrm{Re}_{\mathrm{h}}=30000, \mathrm{Re}_{\theta}=1650$ ). Wall pressure sensors are used to estimate the reattaching location downstream of the step (objective function \#1) and also to measure the wall pressure fluctuation coefficients (objective function \#2). An autonomous multi-variable optimization by genetic algorithm is implemented in an experiment for optimizing simultaneously the voltage amplitude, the burst frequency and the duty-cycle of the high voltage signal producing the surface plasma discharge. The single-objective optimization problems concern alternatively the minimization of the objective function \#1 and the maximization of the objective function \#2. The present paper demonstrates that when coupled with the plasma actuator and the wall pressure sensors, the genetic algorithm can find the optimum forcing conditions in only a few generations. At the end of the iterative search process, the minimum reattaching position is achieved by forcing the flow at the shear layer mode where a large spreading rate is obtained by increasing the periodicity of the vortex street and by enhancing the vortex pairing process. The objective function \#2 is maximized for an actuation at half the shear layer mode. In this specific forcing mode, time-resolved PIV shows that the vortex pairing is reduced and that the strong fluctuations of the wall pressure coefficients result from the periodic passages of flow structures whose size corresponds to the height of the step model.
\end{abstract}

\section{Introduction}

The aeronautic industry faces huge challenges to reach the technical recommendations of national and international advisory research panels. Among them, the reduction in fuel consumption and radiated noise are two recommendations leading to strong expectations for the future, but these recommendations require the definition of innovative techniques. Flow control devices are potential options for simplified aerofoil design and manipulation of turbulent separated flows while maintaining

\footnotetext{
${ }^{*}$ Email address for correspondence: nicolas.benard@univ-poitiers.fr
} 
high aerodynamic performances and low noise emission. Many types of active flow control systems have been defined in the last ten years [1], but depending on the flow conditions they have to be fully optimized in terms of geometry, distribution and operation to reach their better level of control authority. In many situations, the optimal command can be easily defined by in-lab experiments simply by prospecting on the best forcing conditions by a large parametric study. However, when the number of parameters for the command is increasing, a simple parametric study is time-consuming and in most of the case, such an approach is not fully effective. Furthermore, in case of a non-linear flow response with several minima or maxima, simple techniques such as gradient-based approaches can fail to identify a global optimum in particular if the initial point is not well chosen [2-3]. A solution for multi-parameter optimization is the evolutionary algorithm method that has a smaller propensity to converge toward a local optimum when a global optimum is expected, and that can be constructed for solving by experiment multi-parameter research problems as originally defined in

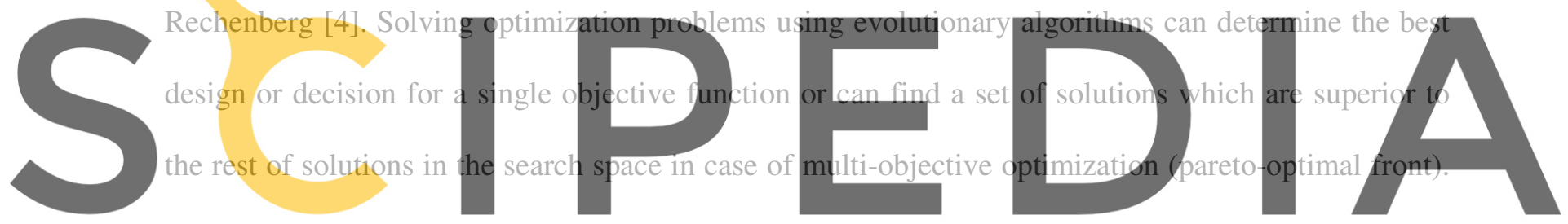

Such a technique is able to find global extremum even in complex functions with many local maxima

Register for free at https//www. s.cipedia.com to download the version without the watermark

instead of experimental analysis software due to their lower cost, lower noise in the data and the complete information on the flow to feed the optimizer. Many studies have been devoted to the optimization of aerofoil shape according to specific needs [5-6], but numerical optimization can also find new relevant applications in the enhancement of active flow control systems. This type of optimization follows the recommendation provided by Gad-El-Hak in its famous book [7] where the author identified evolutionary algorithms as powerful tool for the control of fluid flow. Recently, evolutionary approaches have been implemented in computational fluid dynamics in order to solve multi-criteria optimization problem. These evolutionary algorithms are a stochastic optimization techniques mostly based on the principle of genetics with selection by ranking and design space exploration accomplished by mutation and crossover operators [8]. 
Among several applications, genetic algorithms have been used in combination with CFD for optimizing aerodynamic shapes of high lift multi-elements aerofoil by multi-point adaptation [9-10]. Flow control optimizations have also been reported by combining evolutionary algorithms and numerical simulations. In Hilgers and Boersma [11], the spreading of a turbulent jet is optimized by Direct Numerical Simulation (DNS) regarding the forcing mode and frequency in the initial region of the free shear layer. DNS has also been used for reducing the skin friction on the turbulent wall of a channel flow [12]. The optimal forcing by suction/blowing jet on the upper surface of a NACA0012 aerofoil has been numerically determined in [13]. The problem of time-averaged drag minimization for flow past a circular cylinder has been addressed in different publications [14-15]. Recently, a NSGA-II code has been numerically applied to optimize the flow reattachment along a NACA0015 aerofoil by simple two-dimensional plasma discharge model [16]. In this paper, four design variables and six objectives functions were optimized in order to improve the performances of the aerofoil in

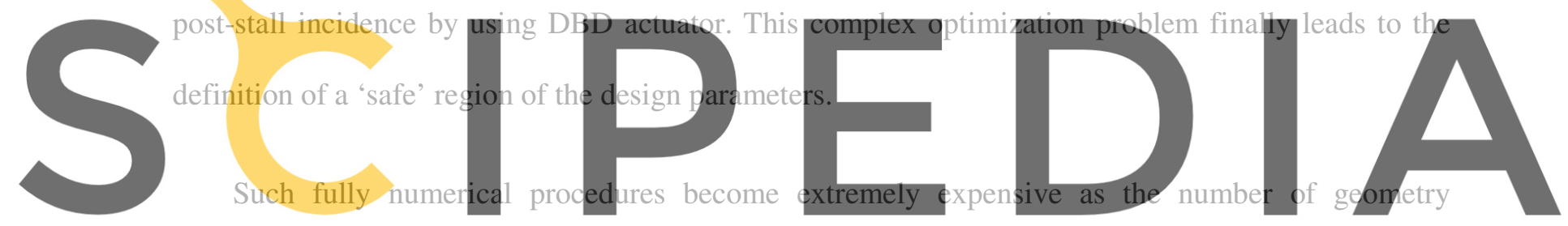

Regisameters is increased. The limitation due to the computational time cost is regularly pointed out, Register for free at https//www.scipedia.com to download the version without the watermark restricting the method to an approximated evaluation of the objective function or limiting the investigations to low Reynolds numbers. In fact, evolutionary algorithms were initially defined to be used in an experimental context [4]. In its earlier applications, the iterative process of optimization by evolutionary algorithm was implemented experimentally by modifying manually the geometry of a two-phase flow model in 1970 [17]. Now, experimental optimization is often referred as Hardware-In the-Loop (HIL) method. Indeed, because evolutionary algorithm only requires the evaluation of the cost function regarding a set of control parameters, such an algorithm can be used in conjunction with experimental measurements. Such a coupling between a numerical solver and wind-tunnel tests has been recently introduced in the experimental flow control community as a convenient way to reach optimal control conditions in autonomous manner (autonomous experimental optimization as named in [18]). Complex application as the experimental design-optimization of a flapping wing (NACA 
0012 equipped with eight actuators to modify its geometry) in order to maximize the produced lift has been proposed in [18]. Active wing morphing has also been investigated in [19] where a two-point optimization design is conducted by interfacing an evolutionary genetic algorithm with a sting balance supporting the wing model in a wind tunnel. Recently, the influence of a flow control system based on dielectric barrier discharge has been optimized by NSGA-II algorithm in a multi-objective optimization approach [20]. Indeed, the autonomous experimental optimization proposed in [20] simultaneously maximizes the lift coefficient estimated by time-averaged pressure measurements and minimizes the electrical power consumed by the actuator. The results are analysed by regarding the trend of the global solutions as well as by inspecting the non-dominated solutions of the multiobjective optimization problem. This elegant approach demonstrates the contribution of each of the design variables on the lift recovery. In [21], the lift production and drag reduction caused by two plasma actuators on the opposite sides of a circular cylinder have been simultaneously optimized

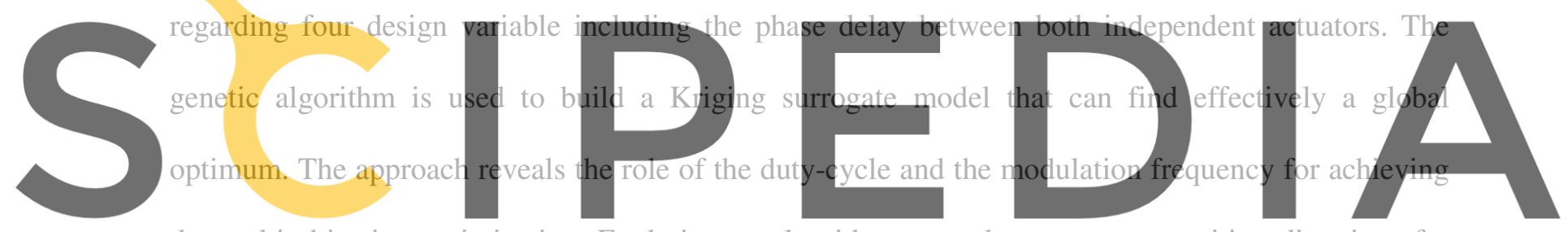

the multi-objective optimization. Evolutionary algorithms can also open new exciting directions for Register for free at https//www scipedia.com to download the version without the watermark obtained by a modei-free approach based on genetic programming methods. This approach, calied machine-learning control, can find a control law depending on the local [22] or global [23] flow conditions in order to minimize or maximize objectives functions selected in regard to the studied case. For instance in [22], the authors find the best control law for minimizing or maximizing the turbulent energy and the spreading of a turbulent mixing layer. The results are compared to an openloop test campaign and confirm the effectiveness of machine-learning to optimize one parameter of the actuator (in the present case the excitation frequency) at a reduced cost time.

The present investigation concerns an experimental turbulent confined flow separation scenario. Indeed, the flow separation downstream of a backward-facing step (BFS) has been selected as the flow configuration to be optimized. This configuration is well-adapted for defining unsteady flow control 
approaches because the flow behind a BFS presents a large variety of periodic motions that can be manipulated. The actuator is based on a surface dielectric barrier discharge [24-26] operated by applying an AC high-voltage to the air-exposed electrode of the actuator as in [20-21]. Plasma actuators are based on electro-mechanical conversion principle that implies fast energy transfers. By adjusting the electrical parameters of the electrical signal applied to the air-exposed electrode, the amplitude, frequency and duty-cycle of the periodic flow fluctuations induced by the plasma discharge can be easily tuned [27] and feed the genetic algorithm optimizer. In the present investigation, a genetic algorithm is used instead of a gradient-based approach simply because the flow response map to a DBD forcing has already been conducted in [28] and it was shown that the flow response is not monotonous as it presents several minima. Furthermore, the proposed method couples a numerical optimizer to a wind-tunnel experiment leading to an autonomous experimental optimization, an approach rarely presented in the recent literature. Single-objective genetic algorithm that solves multi-

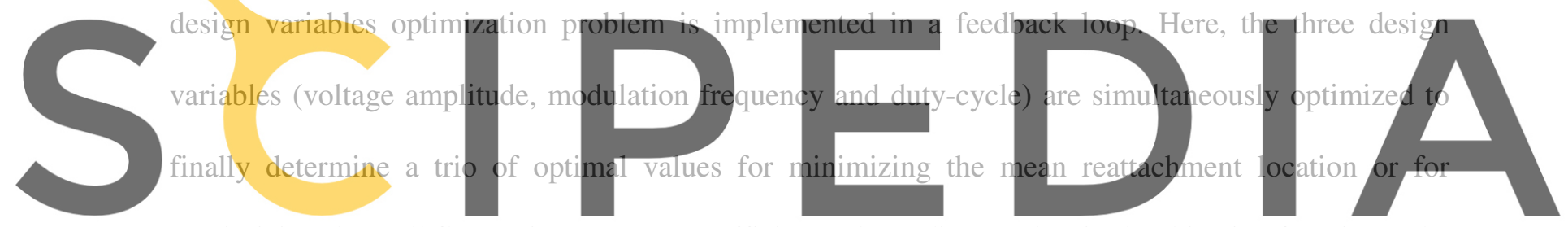

maximizing the wall fluctuating pressure coefficients, depending on the single-objective function to be

Register for free at https//www.scipedia, com to download the version without the watermark

the best parameters identified from the genetic algorithm method in order to highight and detail the physical phenomena behind the optimal forcing conditions.

\section{Experimental setup}

The experiments are conducted in a closed-loop wind-tunnel having a moderate turbulent intensity $(0.8 \%)$. The dimension of the test section is $300 \times 300 \times 1000 \mathrm{~mm}^{3}$. The model covers the full spanwise of the test section and has a height of $30 \mathrm{~mm}$. The aspect ratio (channel width on step height) is larger than 10 to minimize the side-wall influence on the flow in the centre of the wind tunnel. All measurements are performed for a free-stream velocity of $15.6 \mathrm{~m} / \mathrm{s}\left(\operatorname{Re}_{\mathrm{h}}=30000\right)$. A zig-zag turbulator with thickness of $300 \mu \mathrm{m}$ is installed $10 \mathrm{~h}$ upstream of the step corner in order to guarantee a fully turbulent boundary layer at the separation point. In a past experimental campaign [29], the inlet flow 
conditions have been detailed by two-components LDV. At the step corner, the boundary layer thickness is $14 \mathrm{~mm}$ and the momentum thickness is $1.65 \mathrm{~mm}\left(\mathrm{Re}_{\theta}=1650\right)$ for a $\mathrm{H}$ factor of 1.6.

The model has been designed to accommodate the plasma actuator with minimal influence on the incoming boundary layer. The step model includes an internal cavity for placing the grounded electrode while the air-exposed electrode (black-painted aluminium with total thickness of $60 \mu \mathrm{m}$ ) is placed on the opposite side of the model. The air-exposed and the grounded electrode have a width of 15 and $10 \mathrm{~mm}$ respectively and the gap between them is fixed at $2 \mathrm{~mm}$. The model made of PMMA serves as dielectric barrier with a thickness of $3 \mathrm{~mm}$.

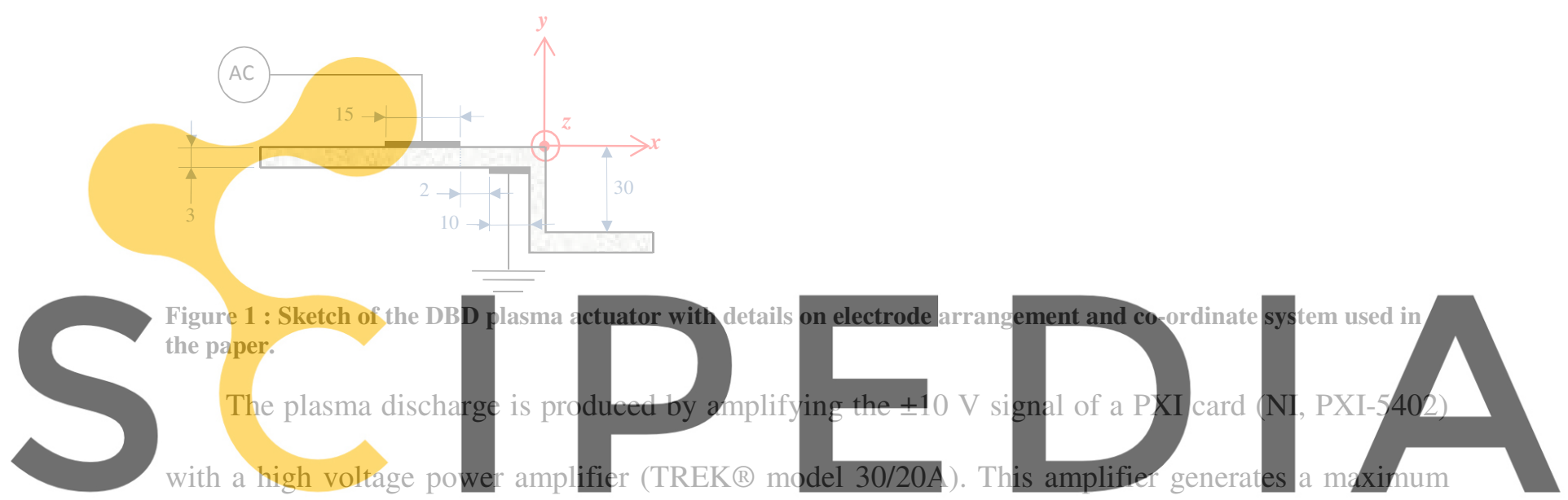

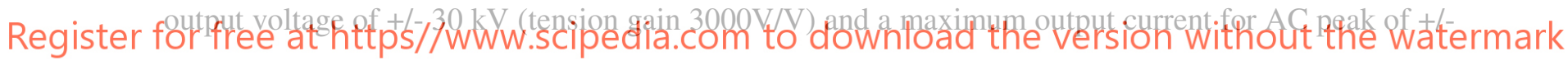

$40 \mathrm{~mA}$ (slew rate 500V/us). The plasma discharge is produced by a sinusoidal waveform with ac

frequency always maintained at $2 \mathrm{kHz}$. Such continuous signal can be effective to manipulate a separated flow, however unsteady forcing by burst modulation is preferred in the present investigation. Thus, the plasma discharge is alternatively turned off and on at frequencies in the range of the periodic fluctuations of the natural flow by using a low frequency gate function to trigger the ac sine voltage (burst modulation, see [26]). Three design variables are simultaneously optimized: the voltage amplitude, the burst frequency and the duty-cycle considering that the driven frequency is fixed (see Figure 2). The voltage amplitude mainly acts on the amplitude of the mean flow produced by the surface discharge. The burst frequency drives the periodic excitation imposed by the discharge. The duty-cycle of the applied signal modifies the amplitude of the flow fluctuations produced by the plasma. High duty-cycle value means that the produced flow approaches steady flow conditions (i.e., 
small fluctuation amplitude) while low duty-cycle will emphasize the amplitude of the fluctuations but in this case the mean produced flow velocity is small [27]. Then, for flow control perspectives, a balance has to be found for producing a 'high' mean flow and one having 'high' fluctuation amplitude. All of these three parameters have strong influence on the performance of the control, this motivating for optimization studies using model free method such as evolutionary algorithm.

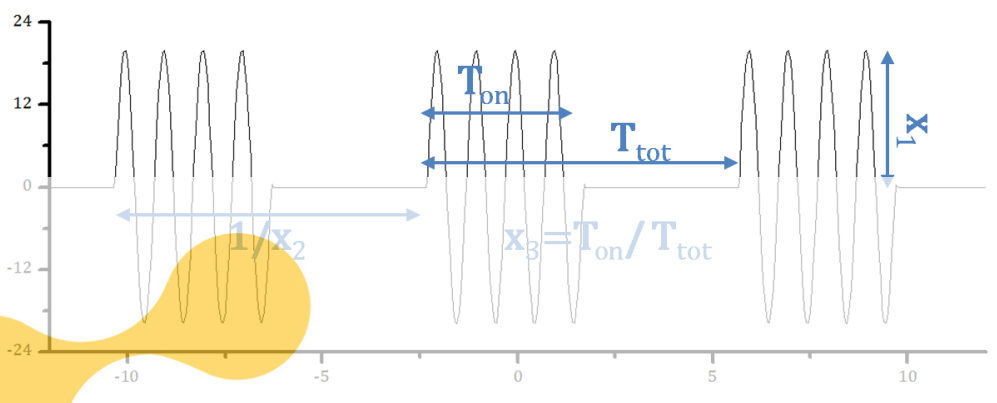

Figure 2 : Illustration of the three design variables simultaneously optimized by the genetic algorithm

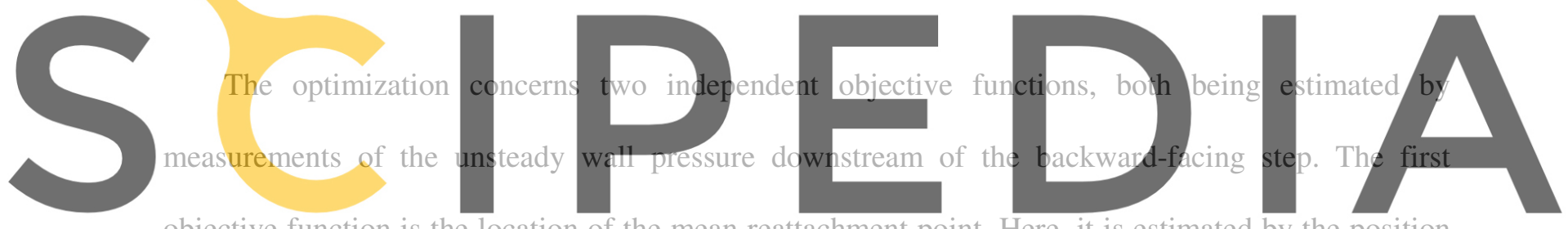

objective function is the location of the mean reattachment point. Here, it is estimated by the position

(Figure 3b). The procedure simply consists in recording the pressure signals on the bottom wall and in computing each local pressure fluctuation coefficients. Then a dedicated gradient-based algorithm seeks for the location of the maximal fluctuation. Determination of the location of maximum $\mathrm{C}_{\mathrm{P}}$, is a convenient and relevant way to estimate the reattachment location without direct measurement $[30,31$, 32], even if it was already observed that such method often leads to a small under-estimation of the real mean reattachment location $[33,34]$. In order to extend the optimization to unsteady component of the flow, a second objective function is considered. The spatial integral of the wall pressure fluctuation coefficient $\mathrm{C}_{\mathrm{P}}$, is performed all along the bottom wall (grey region in Figure $3 \mathrm{~b}$ ). This integral is an estimator of the flow variations in the shear layer developing from the step corner. It is considered that the pressure fluctuations at the bottom wall are caused by the intensity and integral length scale of the vortical flow structures embedded in shear layer and the flapping character of the reattaching flow as it 
is discussed in $[33,35]$. Then, higher value of the $\mathrm{C}_{\mathrm{P}}$, spatial integral is supposed to be related to a new organization or amplification of the unsteady character of the separated flow.

In order to measure the pressure fluctuations downstream of the backward-facing step, the bottom wall has been equipped with 32 pressure taps. These pressure taps consist of holes with $0.6-\mathrm{mm}$ diameter distributed along the streamwise direction from $\mathrm{x} / \mathrm{h}=1$ to $\mathrm{x} / \mathrm{h}=9$. The distance between two successive taps is $4.5 \mathrm{~mm}$ resulting in a spatial resolution of $0.15 \mathrm{~h}$ (see Figure 3a). Unsteady pressure sensors (12 bits) with intrinsic bandwidth of $2 \mathrm{kHz}$ for max pressure of $250 \mathrm{~Pa}$ are used (HCLA 12X5, SensorTechnics, $12.5 \mathrm{kPa}$ dynamic range, $0.6 \mathrm{~Pa}$ of resolution). They are connected to the pressure taps using 350-mm long, 0.8-mm diameter Tygon tubes. The maximum error including non-linearity, hysteresis, and thermal drift is $\pm 2.25 \%$ of the full-scale span. A preliminary dynamic calibration of a single sensor with its tube connection revealed a measurable bandwidth of 6-1200 Hz. Output voltage signals of the pressure sensors are recorded by a multiplexed 32 channels acquisition card (PXI-6259).

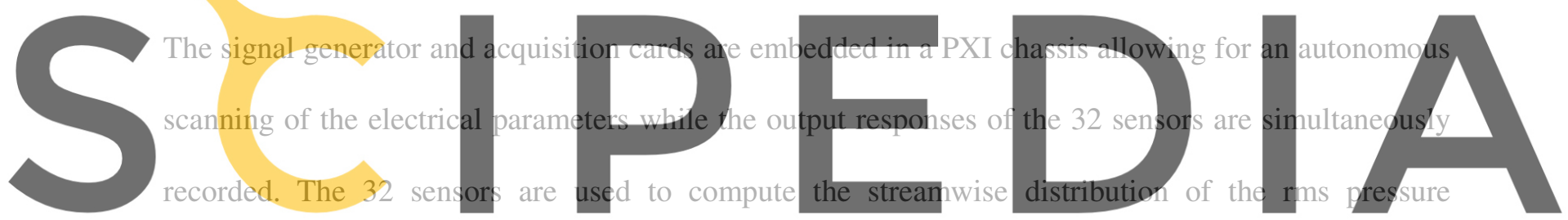

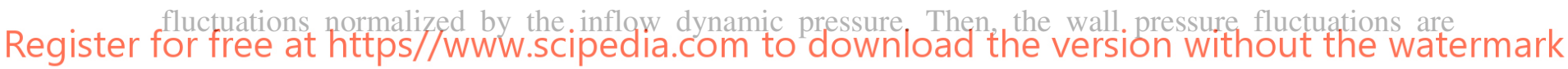
presented in coefficient form:

$$
\mathrm{C}_{\mathrm{P}}=\frac{\sqrt{\frac{1}{n}\left[\left(P_{1}-P_{0}\right)+\left(P_{2}-P_{0}\right)+\cdots+\left(P_{n}-P_{0}\right)\right]}}{0.5 \rho U_{0}^{2}}
$$

where $\mathrm{P}_{0}$ and $\mathrm{U}_{0}$ refers to the reference value of the static pressure and streamwise freestream velocity, respectively. At acquisition rate of $1000 \mathrm{~Hz}$, preliminary tests have shown that a correct estimation of the mean reattachment point requires at least 5 seconds of continuous recording in order to reach a converged mean location. This time is a strong limitation for real-time estimation, but it is intrinsic to the unsteady character of the turbulent reattaching flow in BFS configuration. Another limitation is that the reattachment is estimated within $\pm 0.15 \mathrm{~h}$ resolution due to the spatial resolution of the linear array of pressure sensors. 


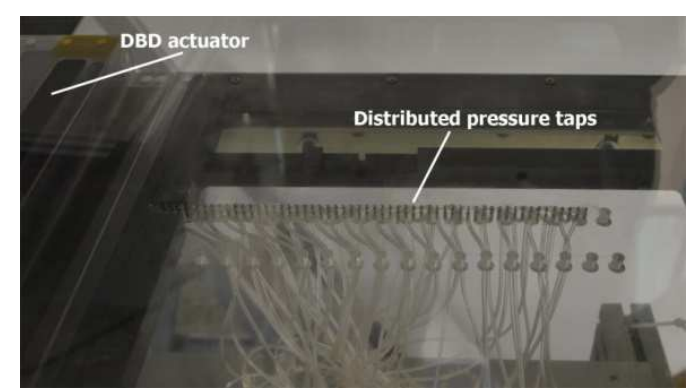

(a)

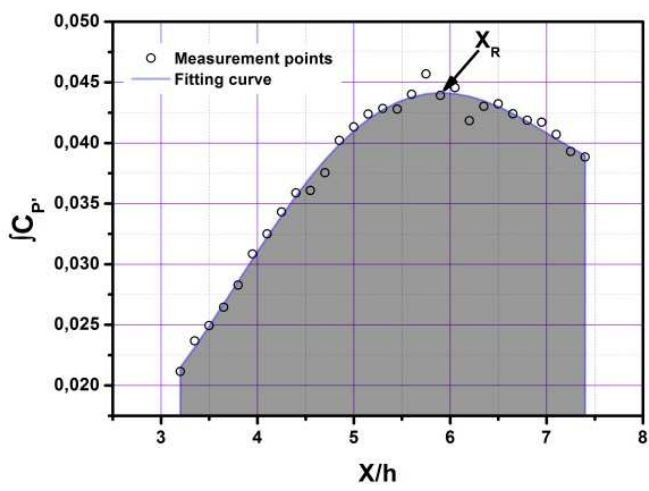

(b)

Figure 3 : View of the pressure taps along the bottom wall (a) and typical streamwise distribution of the wall pressure fluctuation coefficients $C_{P}$, along the bottom wall downstream the step model for $U_{0}=15 \mathrm{~m} / \mathrm{s}(\mathrm{b})$.

Time-resolved particle image velocimetry has been performed for the optimal control parameters identified by the optimizer. The flow is measured by a fast PIV system composed by a high-speed camera (Photron, APX-RS), a single head Nd:YLF high-speed laser with dual oscillator (Quantronix,

Darwin-Duo), a triggering unit (EG, R\&D Vision) and a PC running Davis V8.2 software (Lavision).

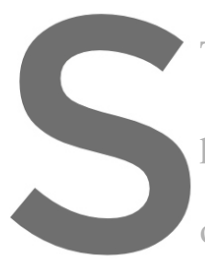

The laser (sheet of $1 \mathrm{~mm}$ ) is placed above the BFS at mid-span of the developing on the side
possibly affected by the boundary layer de
operated at 2000 full frames pen second at $1024 \times 1024$ pixels $^{2}$. The
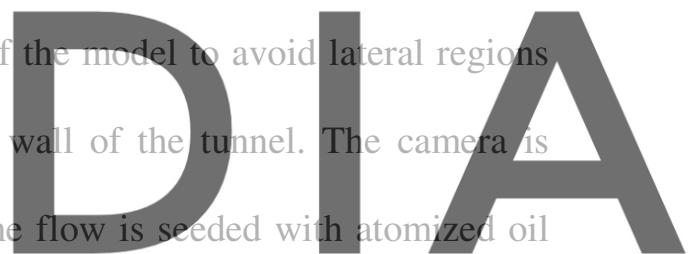

Register (Ondina 15, Shell) resulting in.0.3-0.5 um trackers. Double-frame acquisitions are performed with Register for free at https//www.scipedia.com to download the version without the watermark

frames separated by a time delay of $90 \mu \mathrm{s}$. The two velocity components $(-1<\mathrm{x} / \mathrm{h}<8,-1<\mathrm{y} / \mathrm{h}<1.3)$ are

computed using a cross-correlation algorithm with adaptive multipass, interrogation windows of $64 \mathrm{x}$ 64 to $16 \times 16$ pixels and an overlap set to $50 \%$. This results in a spatial resolution of $2.15 \times 2.15 \mathrm{~mm}^{2}$ for the vector fields. The maximum seeding particle displacement is approximately 8 pixels with a sub-pixel error of \pm 0.1 pixel, that corresponds to a minimal accuracy of $\pm 0.2 \mathrm{~m} / \mathrm{s}$. Flow sequences of 7.5 seconds are recorded. Acquisition starts 10 seconds after the plasma has been turned on, thus the transient regime is discarded from the analysis. The acquisition time is sufficiently long then all the time-averaged quantities (first and second order) have converged to their steady state values (variations lower than 5\%).

\section{Description of the genetic algorithm based optimizer}


Optimization methods define a set of tools and techniques that help engineers to solve complex problems optimizing an objective function while fulfilling a set of constraints. This type of problem can be formulated as follows:

Maximise or minimise an objective function $\mathrm{f}$ depending on $\mathrm{N}_{\mathrm{x}}$ design variables subject to constraints:

$$
g_{j}(x)=0 \text { and } h_{k}(x) \geq 0, j=1, \ldots, N_{g}, k=1, \ldots, N_{h}
$$

where $g_{j}$ and $h_{k}$ are, respectively, the equality constraints with a total number of $\mathrm{N}_{\mathrm{g}}$ and the inequality constraints with a total number of $\mathrm{N}_{\mathrm{h}}$. The variable $\mathrm{x}$ is an $\mathrm{N}_{\mathrm{x}}$ dimensional vector where its arguments are the design variables.

The optimisation problem will result on a set of design variables that defines the Maximum/Minimum value of the objective function. Among the optimization methods available,

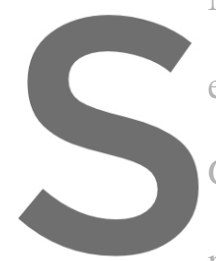
evolutionary techniques and more specifically genetic algorithms are of intenest on this research
Genetic algorithms have evolved quite quiekly thanks to engineering applications. Their stochastic and
robust character makes them very useful in contricx problems where few or none imformation is

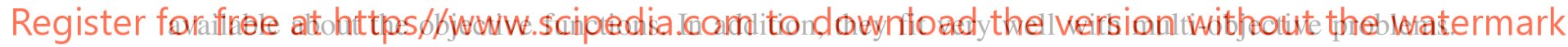

The optimization strategy is inspired on the natural evolution of species, formulated by Darwin. It gets the optimal individuals using a combination of selection, crossover and mutation of the fittest individuals, which are the candidate solutions. The first implementation of GA used a chromosome containing the values of the input variables in the form of a chain binary-encoded, but real-coded genetic algorithms were firstly proposed by Selfridge [36] and later by Bledsoe [37].

The iterative process for optimizing the forcing conditions by a genetic algorithm approach is illustrated in Figure 4. The procedure starts with a randomly generated population (also called generation), including a predefined number of individuals. Here, each generation is composed of ten individuals, one individual being composed of the three design variables introduced previously in the paper. The set of parameters of each individual is applied to the surface plasma discharge and then the 
objective function (or fitness function) is evaluated. The evaluation of one individual takes $5 \mathrm{~s}$ due to the unsteady aspects of the BFS flow. Furthermore, in order to discard transient regimes the pressure acquisition starts 5 seconds after the plasma is turned on. To recover a natural flow regime, a period of 5 seconds is considered after the plasma is turned off. Then, the whole acquisition process takes 15 seconds per individual plus a few hundred of microseconds to read and write the input and output ASCII files. At the end of the evaluation process all individuals can be ranked according to the value of their fitness function. At this time, the basic operators which manage the genetic algorithm, namely the selection, the crossover and the mutation, are applied. The selection operator takes care of selecting the best performing individuals of each population to enable other operators to produce new offspring. It is the most basic operator and usually the first one to be applied. The most usual selection techniques are roulette wheel, ranking selection, and Elitism, among others. In the present investigation, the best individuals are selected by a ranking tournament. Crossover operator takes two selected individuals and it combines them to create a new offspring. Several techniques can be used to determine the point where the genetic information is split to add the genetic information from the second individual. Here, single-point crossover, the most standard techniques for crossover, is applied (probability of 0.9). Finally, the mutation operator creates new offspring modifying the information contained in one design variable of a selected individual. Bit inversion and order changing are usual mutation techniques for binary encoding, but here (because of real-value encoding approach) a small quantity is added to the design variable under mutation process. Furthermore, the mutation probability is set as a function of the number of design variable; it is defined as $1 / 3$ because three designs variables are considered. At the end of the operator procedure, a new generation is produced and the iterative process starts. This iterative process finish when the stopping criterion is reached (it could be accuracy, time, number of iterations, etc...). Here, the genetic approach tests a total of 12 generations for a total running time of 45 minutes. The procedure is fully automatized and it corresponds to a closed-loop approach by autonomous experiment optimization.

The selected optimizer used in this work is the Single-Objective Genetic Algorithm (SOGA) module in Robust Multi-objective Optimization Platform (RMOP) developed at CIMNE. Details of 
RMOP can be found in references [38, 39]. This optimization platform enables the analysis of single and multi-objective problems using the hybridized techniques that combine Pareto-game and Nashgames. However, in this work only the subset of tools corresponding to single objective optimization problems has been used and this simplified use of the optimization code is not using the parallel computing capabilities in order to avoid overloading the plasma actuator with more than one individual at the same time.

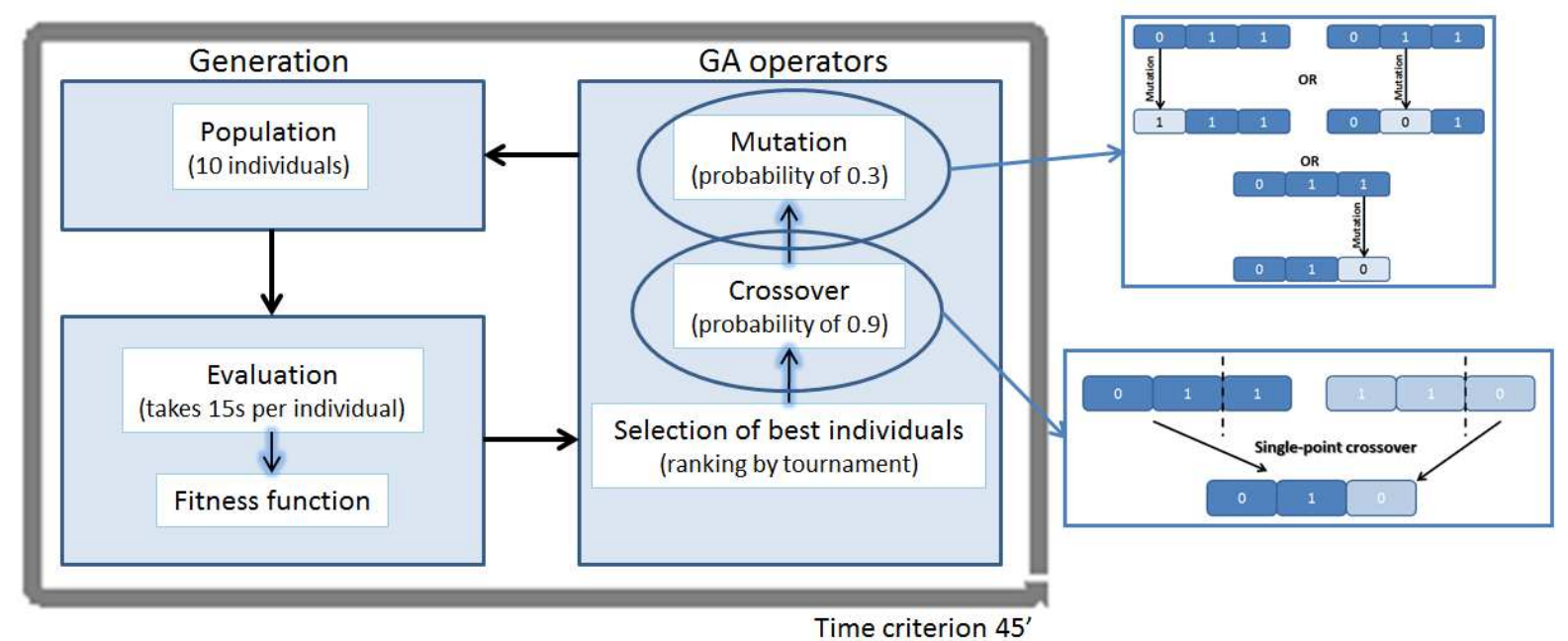

Figure 4 : Illustration of genetic algorithm principle

\section{Results - Optimization by GA method}

The procedure of coupling experimental sensors and actuators with a numerical optimization code, as an analogic solver, has been described in the previous sections of this paper. Now, the results of the optimization are presented regarding each of the objective functions (reattachment minimization or wall fluctuating pressure maximization).

\section{IV-1 Minimization of the reattachment location}

In this first optimization case, the reattachment length $X_{R}$ is the objective function of the optimization problem. The objective is then:

Minimize

$$
f_{o}(\overrightarrow{\boldsymbol{x}})=\min \left[X_{R}(\overrightarrow{\boldsymbol{x}})\right]
$$


where $\overrightarrow{\boldsymbol{x}}$ is the vector containing the three design variables $\left(\boldsymbol{x}_{1}, \boldsymbol{x}_{2}, \boldsymbol{x}_{3}\right)$ of the DBD plasma actuator.

The optimization problem, equation (3), is defined as a single-objective minimization problem, with the following definition of the lower-upper bounds search space of the three design parameters:

- $\quad x_{1}$ : Voltage amplitude $(\mathrm{kV}) ; \mathrm{V} \in \mathbb{N}, 12 \leq \mathrm{V} \leq 20$

- $\quad \boldsymbol{x}_{2}$ : Burst Frequency $\left(f_{b m}\right.$, in $\left.\mathrm{Hz}\right) ; f_{b m} \in \mathbb{N}, 10 \leq f_{b m} \leq 300$

- $\quad \boldsymbol{x}_{3}$ : Duty Cycle (DC, in \%); DC $\in \mathbb{N}, 5 \leq \mathrm{DC} \leq 95$

Some of the evaluations of the populations are illustrated in Figure 5. This plot shows the optimization process for the three design parameters. The first generation is produced randomly and then it covers a large search space region. However, regardless of the applied electrical parameters, all the evaluations indicate a reduction of the recirculating area when the surface plasma discharge operates. The convergence of the iterative process is clearly evidenced in Figure 5. For instance, the design variable corresponding to the voltage amplitude reaches its final value in only a few generations (see generation 7 in Figure 5Erreur! Source du renvoi introuvable.), but other parameters such as the burst frequency need more time to converge. The mutation of the individuals slows the convergence rate but also insures that the best solution is found even in context of several local minima in the static response of the flow. 

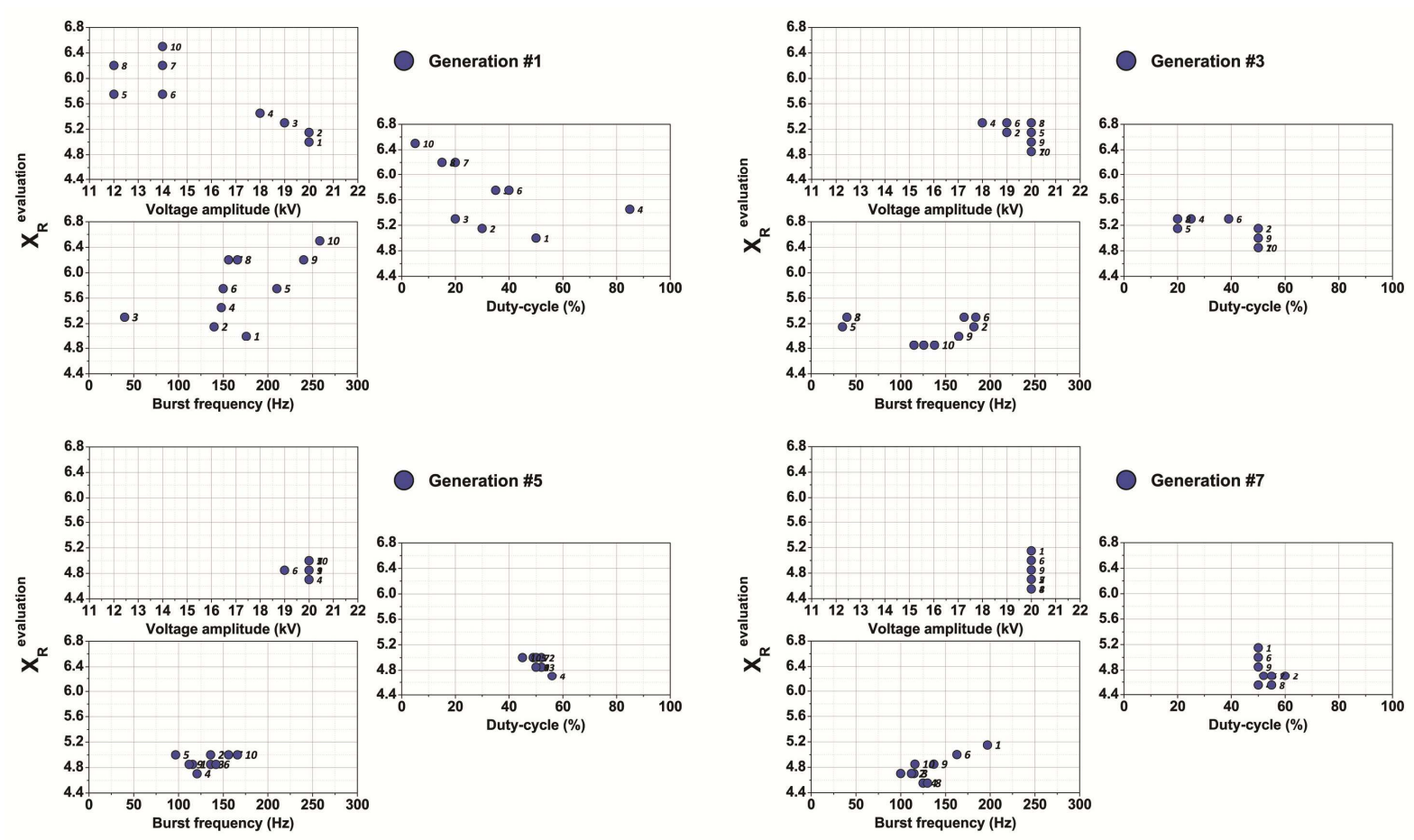

Figure 5 : Evaluation of the individuals of different generations for the reattachment optimization by SOGA.

The convergence of the best solution of each generation is shown in Figure 6. This plot shows how fast the convergence of the optimization is. After 60 evaluations (i.e., six generations), the minimized reattachment length is $4.55 \mathrm{~h}$ meaning that the recirculation bubble has been reduced by $20 \%$. This reduction is in agreement with [29] but larger reductions by using surface plasma actuators are reported in [40] or [41] in case of an initial laminar flow regime. The reduction in size of the recirculating flow is also similar to those obtained at similar $\mathrm{Re}_{\mathrm{h}}$ by using an oscillatory jet with a continuous slit exhaust [42] or an oscillatory flap [31]. At the end of the iterative optimization process, the best individual find by the solver corresponds to periodic forcing with voltage amplitude at its maximal value (i.e., $20 \mathrm{kV}$ here), a duty-cycle in the 50-60\% range and superimposed perturbations in a $120-130 \mathrm{~Hz}$ range. As it is shown in Figure 7 where the distribution of the design variables of the 120 individuals are plotted, many of the individuals have design variable values around the optimal forcing condition due to the fast convergence rate of the method. The most effective periodic forcing frequency when scaled on the height of the step or the initial momentum thickness leads to Strouhal numbers of about $\mathrm{St}_{\mathrm{h}}=0.23$ and $\mathrm{St}_{\theta}=0.013$, respectively. Periodic forcing at these Strouhal numbers has been largely documented in literature for other types of flow control devices than the surface plasma 
discharge used in the present investigation [30, 31, 32, 33, 34]. For instance, Chun and Sung in [42] identified the optimal local forcing at $\mathrm{St}_{\mathrm{h}}=0.27\left(\mathrm{Re}_{\theta}=1470\right.$, pulsed jets produced by loud speakers $)$. In [43], Bhattacharjee et al. identify a $0.2-0.4$ Strouhal range for which loud speaker installed on the top wall of the test section can minimize the recirculation bubble. By large eddy simulation, Merhez et al. [44] shows that the recirculating flow is reduced for sinusoidal oscillating jet positioned at the step edge and operated at $\mathrm{St}_{\mathrm{h}}=0.25$. A similar optimum frequency can be found in the wake of bluff body as it is shown in [45]. In fact, the separated shear layer formed downstream of a BFS evolves as a typical mixing layer in its earlier stage of formation with a typical 'shear layer mode' of instability at about $\mathrm{St}_{\theta}=0.012$ (where $\theta$ is the momentum thickness of the boundary layer) [46]. Forcing a separated shear layer with periodic perturbations at $\mathrm{St}_{\theta}=0.013$ is well known to promote a faster growing of the shear layer by an increase of the pairing mechanism [47]. This type of forcing corresponds to a manipulation of the shear layer mode of instability [48] for which a larger spreading rate results in a shortened reattachment.
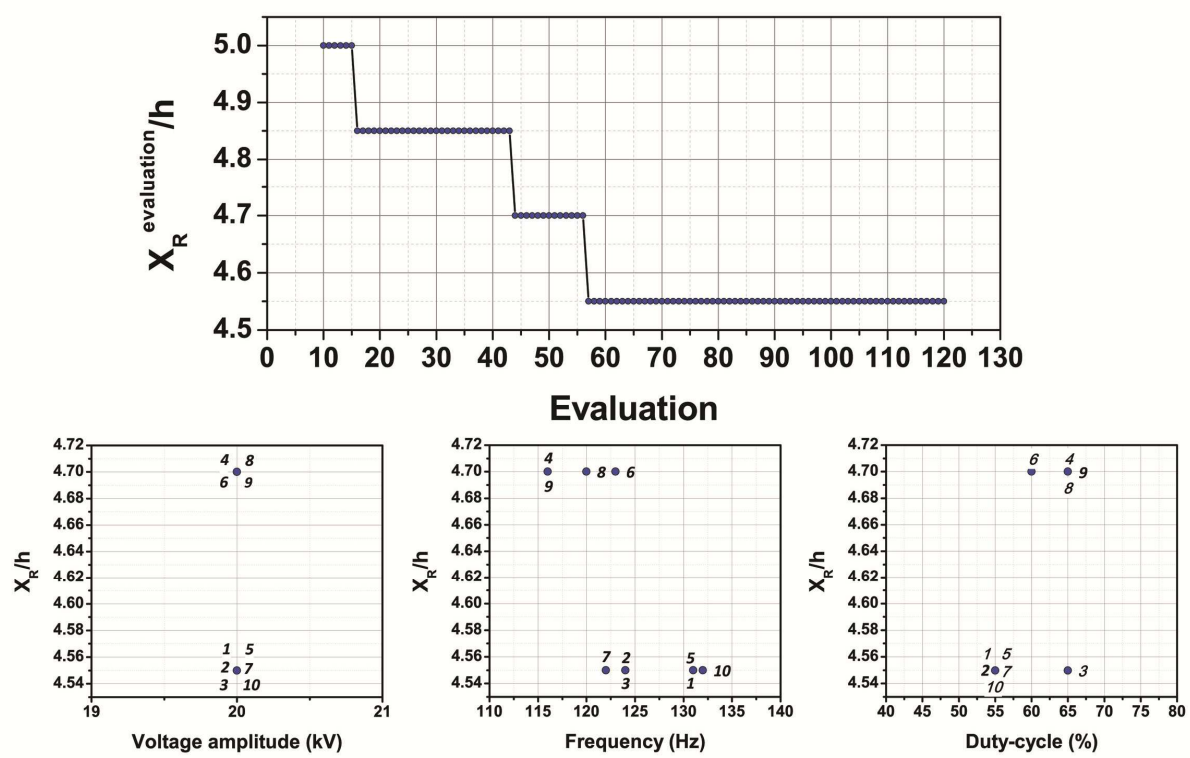

Figure 6 : Convergence of the best individual of one generation (top) and fitness function of the final generation (\#12) according to the voltage amplitude, the burst frequency and the duty-cycle. 

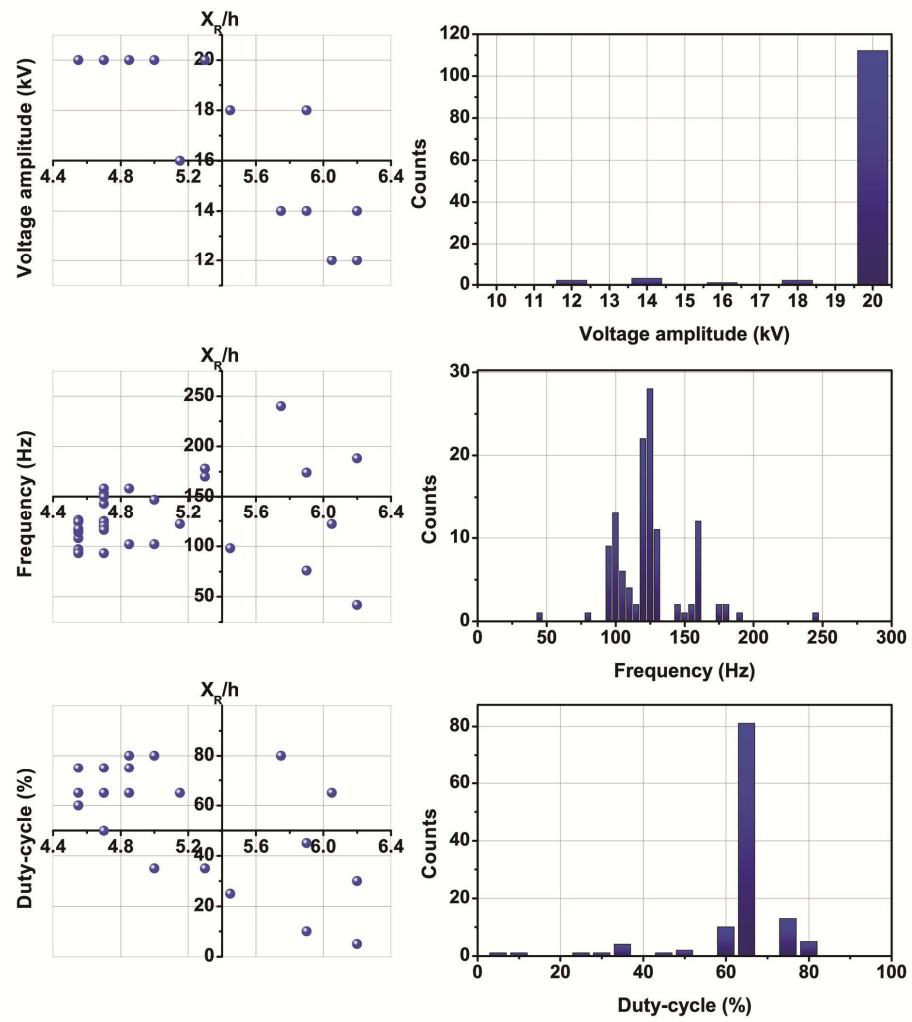

Figure 7 : Evaluations of the total populations autonomously generated by the genetic algorithm.

IV-2 Maximization of the wall pressure fluctuations

The second optimization test case problem selects the $\mathrm{C}_{\mathrm{P}}$, spatial integral value as the objective function to be improved. Thus, the optimization problem is now defined as:

Maximize

$$
f_{o}(\overrightarrow{\boldsymbol{x}})=\max \left[\int C_{P},(\overrightarrow{\boldsymbol{x}})\right]
$$

where $\overrightarrow{\boldsymbol{x}}$ is the vector containing the three design parameters of the plasma DBD actuator and the integral symbol is the spatial integration from $\mathrm{x} / \mathrm{h}=3.2$ to $\mathrm{x} / \mathrm{h}=7.4$.

Again, the optimization corresponds to a single-objective maximization problem with multi design variables. These variables have the same lower-upper bounds search space than in section IV-1. 

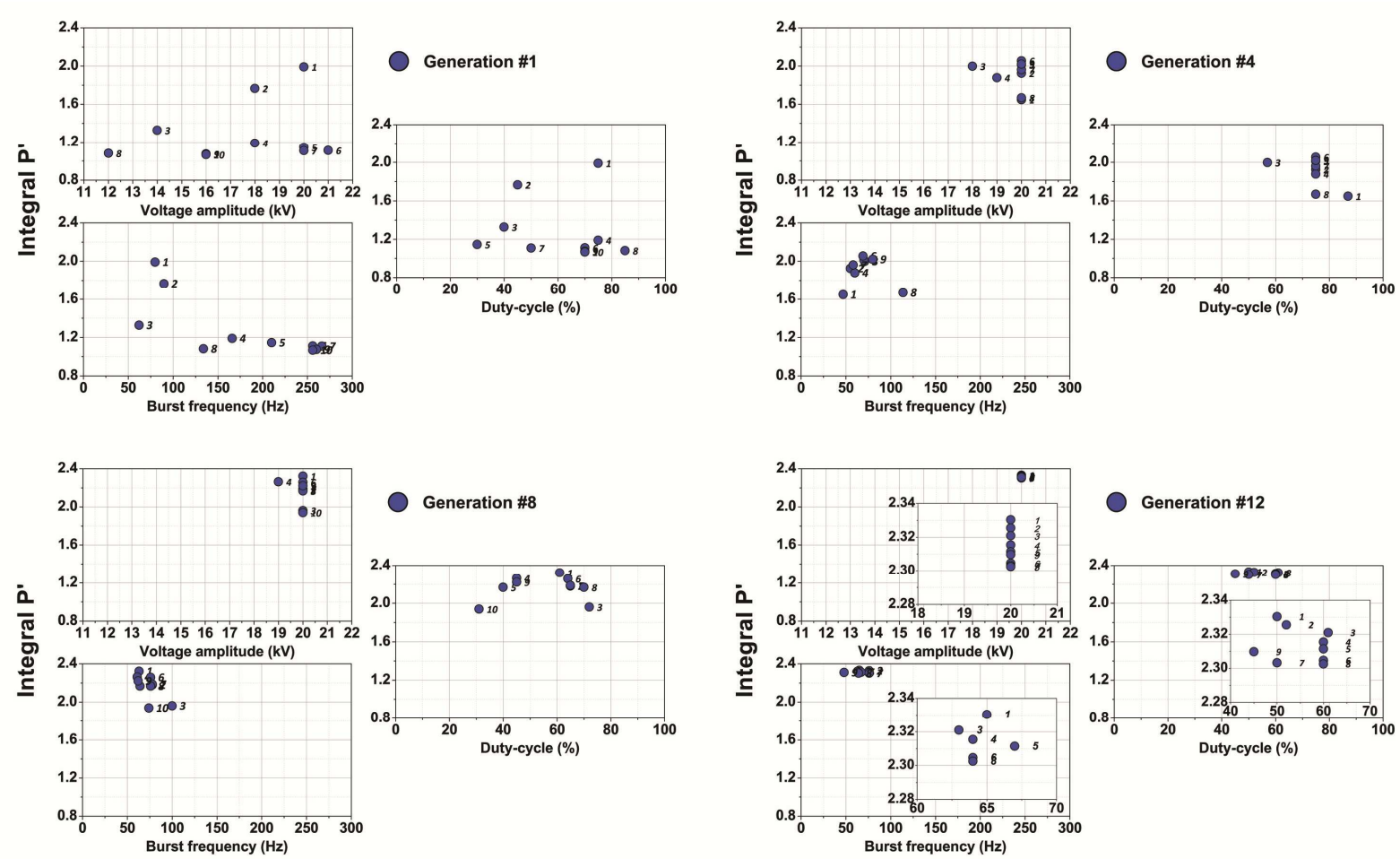

Figure 8 : Four generations of the maximization of the wall fluctuating pressure coefficients

The evolution of the individuals of a few of the tested populations is shown in Figure 8. Starting from a population of random individuals (generation 1), the electrical parameters finally collapse to values covering a tiny range. At the end of the iterative process, the GA code identifies the best forcing conditions that maximize the wall fluctuating pressure. For periodic forcing with maximal voltage amplitude, duty-cycle of $50 \%$ and perturbation frequency at $65 \mathrm{~Hz}$, the wall fluctuating pressure coefficients are maximized with an increase by $115 \%$ by comparison with the non-forced flow conditions. The convergence rate of the SOGA approach is lower than the one for the objective function related to the reattachment location, here variables of the best candidate have been found for the last generation (Figure 9). The optimal voltage amplitude is found from the first generations because this variable has a strong influence on the control authority (in fact, it drives the amplitude of the mean flow produced by the discharge). However, the convergence is slowed by the definition of the optimal duty-cycle and in a lesser extent by the determination of the best forcing frequency. As it is presented in Figure 9, the individuals of the total population present frequency variable within a 50$100 \mathrm{~Hz}$ range and duty-cycle covering a 40-80\% range. Here, the optimal forcing frequency corresponds to a Strouhal number $\mathrm{St}_{\mathrm{h}}=0.125\left(\mathrm{St}_{\theta}=0.006\right)$, but also to a Strouhal number based on the 
reattachment location equal to $\mathrm{St}_{\mathrm{XR}}=0.6$. This frequency corresponds thus to the frequency of the vortical flow structures when they impact the bottom wall [30, 35, 47]. That suggests that the best forcing frequency for maximizing the wall pressure fluctuations matches with the one at the end of the shear layer development when the dividing line curves to the bottom wall. This mode of forcing corresponds to the first subharmonic of the shear layer mode. Kaul recently confirmed that the first subharmonic of an instability wave is a dominant parameter in the development and growth rate of a separated shear layer [49]. However, single-frequency forcing at the subharmonic has only been investigated in a few publications but, in most cases, this has been conducted in a general context of double-frequency excitation where forcing at the fundamental and its subharmonics are considered simultaneously or with a phase delay $[50,51,52]$. Because double-frequency excitation is more effective than single-frequency due to subharmonic resonance $[53,54,55]$, the case of singlefrequency excitation at the subharmonic has not been fully documented in these publications. Here, the GA method has identified such a forcing mode as the more effective for maximizing the wall pressure fluctuations in case of BFS flow configuration. This specific forcing condition will be detailed later in the paper. 

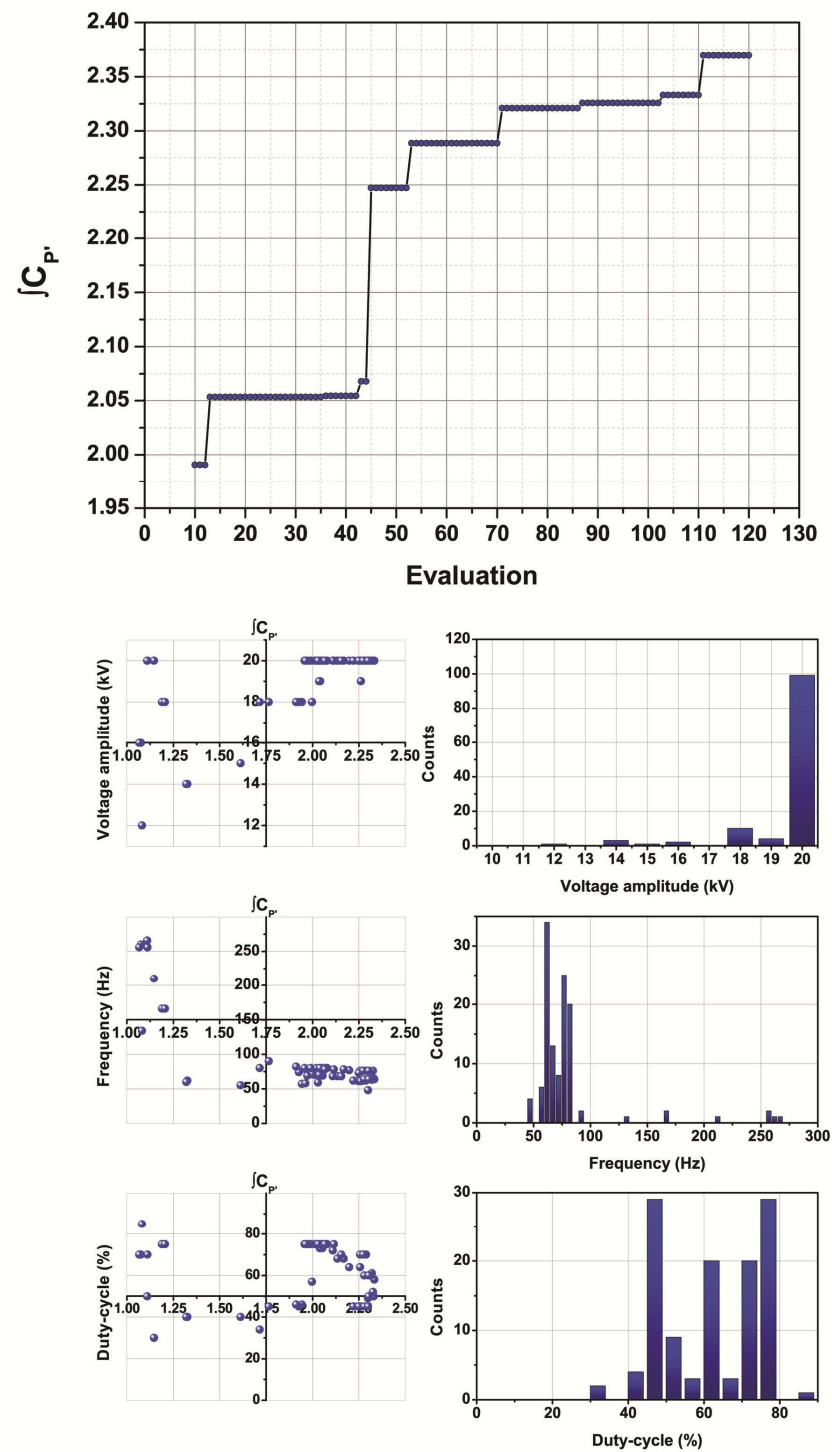

Figure 9: Convergence of the best individual for $P^{\prime}$ integral value and evaluations of the complete population of 120 individuals.

\section{Results - PIV measurements on the best forcing conditions identified by SOGA}

The optimal design variables for maximizing the pressure fluctuations or minimizing the reattachment location have been identified by the GA optimizer in the previous part. Now, the flow response to the trio of optimized parameters is detailed by a time-resolved PIV analysis.

\section{V-1 Mean flow quantities}

The mean velocity field normalized by the freestream velocity $\mathrm{U}_{0}$ is plotted in Figure 10 for the natural flow case as well as the two combinations of parameters defined from the GA code. The iso-contours or the representations by streamlines confirm that the actuation leads to a reduction of the recirculating 
bubbles for both applied signals. From these data, the location of the reattachment point is determined as the point where the velocity $\mathrm{U}$ is equal to zero at a distance of $0.05 \mathrm{~h}$ from the bottom wall. The natural flow reattaches at $5.83 \mathrm{~h}$ downstream of the step wall. This value corroborates with the reattachment location extracted from the wall pressure fluctuations distribution. When the plasma discharge is operated at $20 \mathrm{kV}, 65 \mathrm{~Hz}$ and $50 \%$ of duty-cycle $\left(\mathrm{St}_{\theta} \approx 0.06\right.$, Figure $\left.10 \mathrm{~B}\right)$, the mean reattachment point is moved further upstream with a location identified at a distance of $5.12 \mathrm{~h}$ from the step. For the design variables optimizing the reattachment location $\left(\mathrm{St}_{\theta} \approx 0.013\right.$ from the SOGA code, Figure 10C), the mean flow reattaches at $4.55 \mathrm{~h}$. The flow measurements confirm the $22 \%$ reduction in the reattachment length due to the DBD actuator.

The modifications caused by periodic forcing are further detailed by inspecting the evolution of the vorticity thickness with downstream location $\mathrm{x} / \mathrm{h}$ (Figure 11). At the separating point located at the step corner, the vorticity thickness is small but it rapidly grows with the downstream distance. Regardless the presence of forcing conditions or not, a linear growing is observed up to $\mathrm{x} / \mathrm{h}=3.5$ (zone I in Figure 11). Beyond this location, the vorticity thickness of the natural flow stops its development from $\mathrm{x} / \mathrm{h} \approx 3.5$ up to $\mathrm{x} / \mathrm{h} \approx 5.5$. The stabilization of the vorticity thickness (zone II) should result from the completion of the merging process or because the vortices stop their development in size [56]. When subharmonics forcing is performed at $\mathrm{St}_{\theta}=0.006$, the shear layer stops its linear development at an upstream position (at $\mathrm{x} / \mathrm{h} \approx 4$ ) and the vorticity thickness is maintained at a constant value up to $\mathrm{x} / \mathrm{h} \approx 5$. The major modifications in zone II are observed when the plasma is operated at $\mathrm{St}_{\theta}=0.013$. In this latter case, the shear layer stops its development at a shortened distance $(\mathrm{x} / \mathrm{h}=3)$ while downstream of this location (zone II) the vorticity thickness still increases but with a reduced rate. It is assumed that the reduced spreading rate of the shear layer caused by the discharge in zone II corresponds to a change in the growing mechanism of the fully developed shear layer compared to zone I. The amalgamation of adjacent vortices contributes to a larger spreading of the shear layer but the pairing of vortical flow structures is not the only mechanism to promote the divergence of a turbulent shear layer. For instance, a smaller spreading rate has been observed in the process of vortex splitting than in vortex merging [57]. Furthermore, the shear layer growing can also be caused by the growth of eddies 
by fluid entrainment and not to the pairing mechanism as shown in Brown and Roshko [58].

Unfortunately, an analysis based only on mean flow quantities cannot reveal the real dynamic corresponding to the flow in zone II. Beyond the reattachment point (end of zone II), in all cases the vorticity thickness increases again due to the lateral displacement of the reattaching region (zone III).
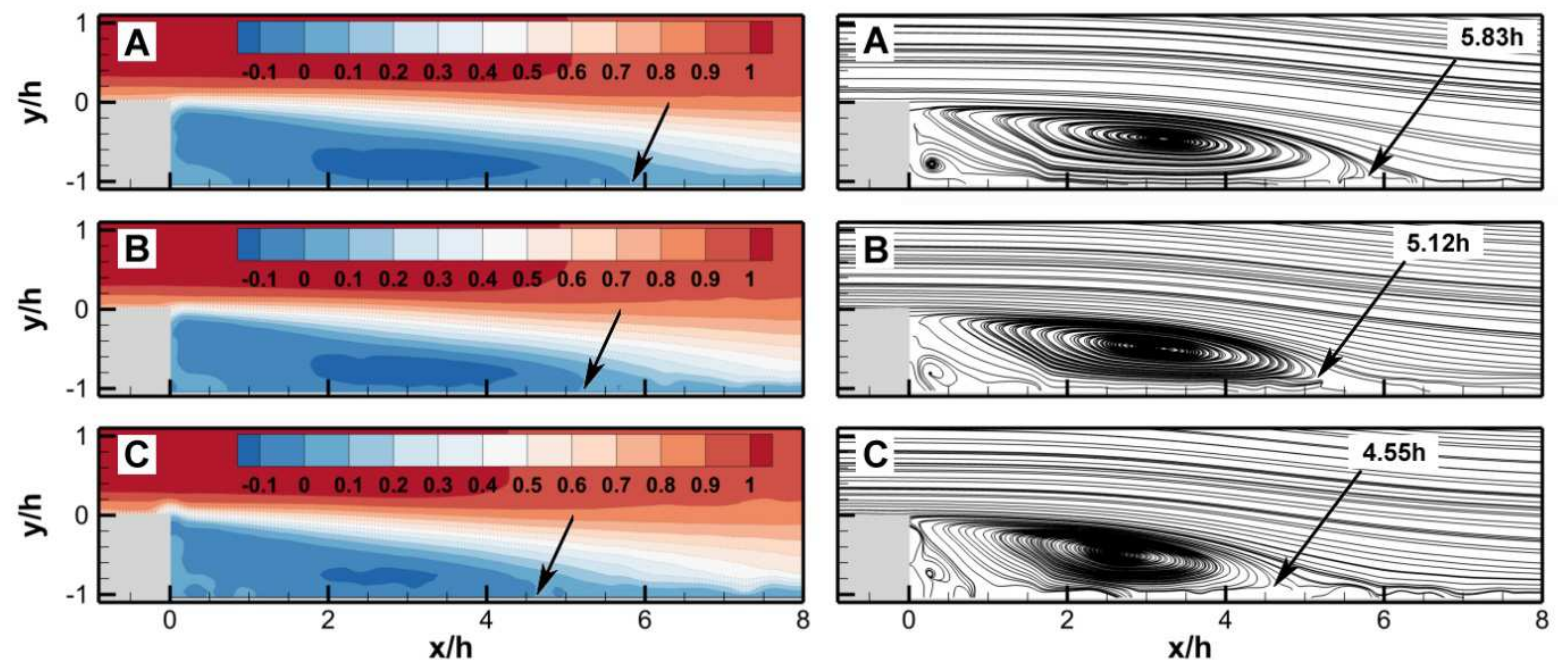

Figure 10 : Mean velocity field $U$ normalized by $U_{0}$ (left plot), and the streamlines of the flow for the two optimal sets of design variables (right plot). The baseline flow is presented in (a), the actuation at $\mathrm{St}_{\mathbf{\theta}}=0.006$ in (b) and plasma actuation at $\mathrm{St}_{\mathbf{\theta}}=\mathbf{0 . 0 1 3}$ in (c). 

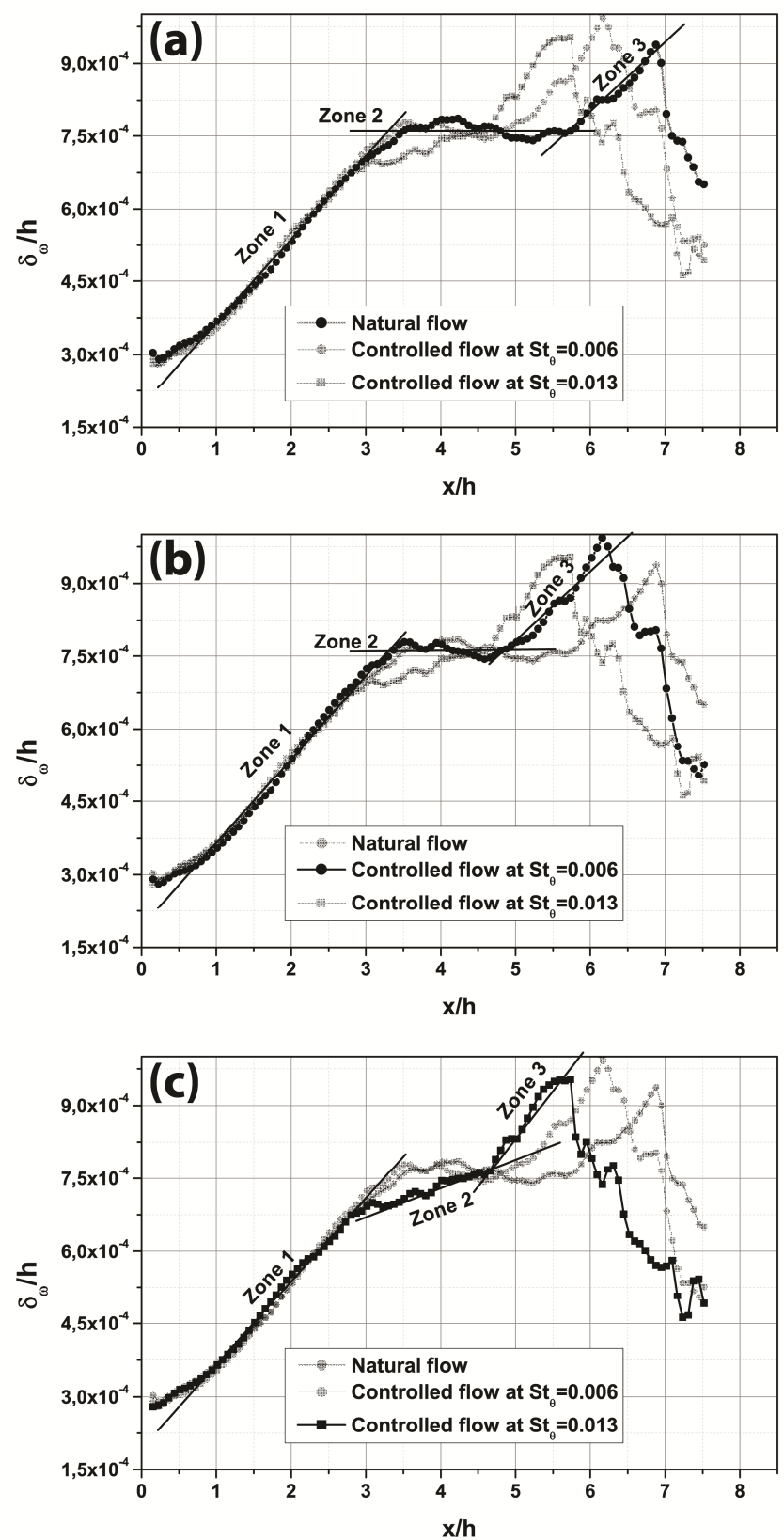

Figure 11 : Evolution of the vorticity thickness with downstream location $\mathrm{x} / \mathrm{h}$ for natural $(\mathrm{a}), \mathrm{St}_{\theta}=0.006(\mathrm{~b})$ and $\mathrm{St}_{\theta}=\mathbf{0 . 0 1 3}(\mathrm{c})$.

\section{V-2 Time-resolved analysis of the turbulent separated flow}

Here, the time-resolved fluctuating velocity v' is extracted from the PIV fields at $y / h=0.05$ in order to analyse the temporal evolution of the separated shear layer. The presence and convection of the vortical flow structures is clearly evidenced by the alternations of positive and negative v' velocity components and also by the orientation of the streaks shown in Figure 12. The convection velocity, $U_{c}$, is $0.55 \mathrm{U}_{0}$ for the natural flow and it is slightly reduced to $0.5 \mathrm{U}_{0}$ when the plasma discharge is applied. However, the main result from this figure is the strong regularization of the vortical flow structures of 
the shear layer for a forcing at the shear layer mode $\mathrm{St}_{\theta}=0.013$. It is shown in Figure $12 \mathrm{c}$ that the flow quickly organizes in a periodic manner (from $\mathrm{x} / \mathrm{h}=1$ ) and it exhibits a strong periodization between $\mathrm{x} / \mathrm{h}=2$ and $\mathrm{x} / \mathrm{h}=4$. Furthermore, the frequency signature of the vortex street is halved past the $\mathrm{x} / \mathrm{h}=4$ position probably due by the vortex pairing. An actuation at $\mathrm{St}_{\theta} \approx 0.006$ (Figure $12 \mathrm{~b}$ ) also affects the flow dynamics, but its influence is not as clear as the regularization imposed by forcing at the shear layer mode. The amplitude of the fluctuating v' velocity is clearly reinforced, even more than forcing at $\mathrm{St}_{\theta}=0.013$, but the shedding regularization is less pronounced. The flow structures increase in size when they are convected, but their mechanism of growing is not clear from Figure 12b. The extraction lines confirm the presence of oscillations in the flow with a wavelength increasing along the vortex street development as it was observed for the natural flow.
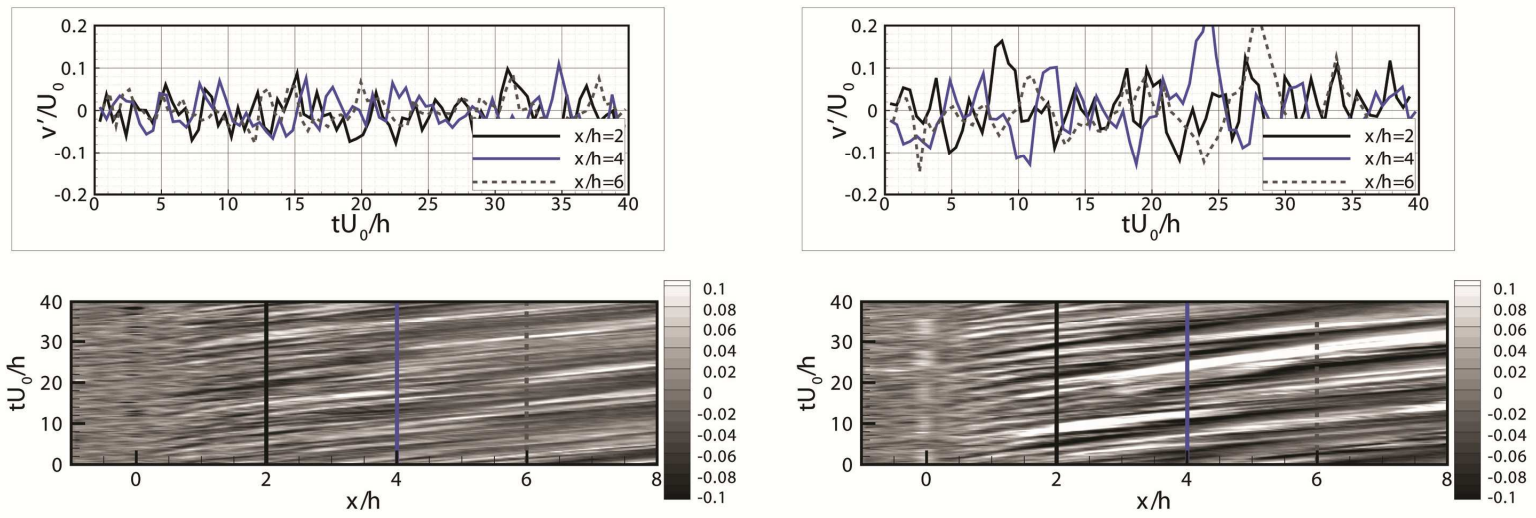

(a)

(b)
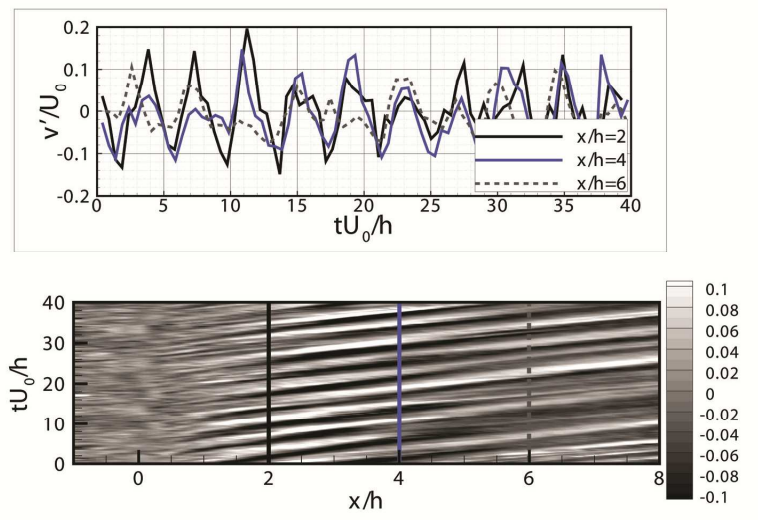

(c)

Figure 12 : Time evolution of the fluctuating velocity $v^{\prime}($ at $y / h=0)$ along the bottom wall and zoomed views at $x / h=2,4$ and 6 for the natural flow (a), periodic forcing at $\mathrm{St}_{\theta}=0.006$ (b) and $\mathrm{St}_{\theta}=0.013$ (c). 
Complementary to the analysis done over a short flow sequence as in Figure 12, the power density spectra of the fluctuating component v' at three locations along the shear layer mean centerline are shown in Figure 13 by considering each complete series of flow measurements. At the beginning of the shear layer development $(\mathrm{x} / \mathrm{h}=2)$, the turbulent energy of the baseline flow is small and no discrete frequency dominates. The periodic behavior of the shear layer is not fully installed at this location. Beyond this position, the turbulent energy is increased, but the frequency content of the turbulent flow is spread over a wide frequency band with maximal amplitude of the PSD observed at low frequency ( $\sim 7 \mathrm{~Hz}$, presumably the physical frequency related to the flapping of the shear layer). A small amplitude bump can also be observed at about $72 \mathrm{~Hz}$ and its first subharmonics, the signature of periodic events at these frequencies.

For flow forcing at $\mathrm{St}_{\theta}=0.006$, the shear layer presents periodic components from the earlier stage of the shear layer development $(\mathrm{x} / \mathrm{h}=2)$ as demonstrated by the small amplitude peak at the forcing frequency and its two first harmonics in the PSD. Downstream of this position, a strong and sharp peak is observed at the forcing frequency, while only a small signature of vortex pairing is visible at $\mathrm{St}_{\theta} / 2$. The signature at the forcing frequency is continuously amplified all along the shear development, this even downstream of the mean flow reattachment. As it was discussed in [56], the amplification of the subharmonic component occurs over a longer distance by comparison with the amplification of the fundamental. Here it is demonstrated that the subharmonic component is continuously amplified up to the reattaching region. This constant increase with only a small signature of pairing activity and the enlargement of the streaks in Figure 12 indicate that the growing mechanism of the coherent flow structures is primarily due to an entrainment process. This is further confirmed by the time-resolved velocity flow fields depicted in Figure 14. By plotting the vector field in a zoomed view together with $\Gamma_{2}$ criterion as it is defined by Graftieaux et al. [59], this figure shows how the vortical flow structures predominantly evolves when they are convected downstream of the step. A visual inspection of the vector fields shows that vortex pairing occurs at some instants of the flow sequence however the most recurrent scenario is a growing of the vortical flow structures by entrainment of the fluid when they are convected. As it is shown in Figure 14, the vortical flow structures are already formed at $\mathrm{x} / \mathrm{h}=1$. They are stretched during their convection but they remain 
coherent all along the shear layer development. In its final stage, the flow structure has a size corresponding to the step height. Thus, the upper part of the vortical flow structure is in direct interaction with the main flow velocity (i.e., the flow in the upper part of the shear layer) that helps the flow structure to gain rotation speed and finally promotes a low resident time in the mean recirculating region. These flow structures are periodically shed in the wake of the BFS, this shedding being responsible for the strong frequency signature in the PSD of Figure 13.

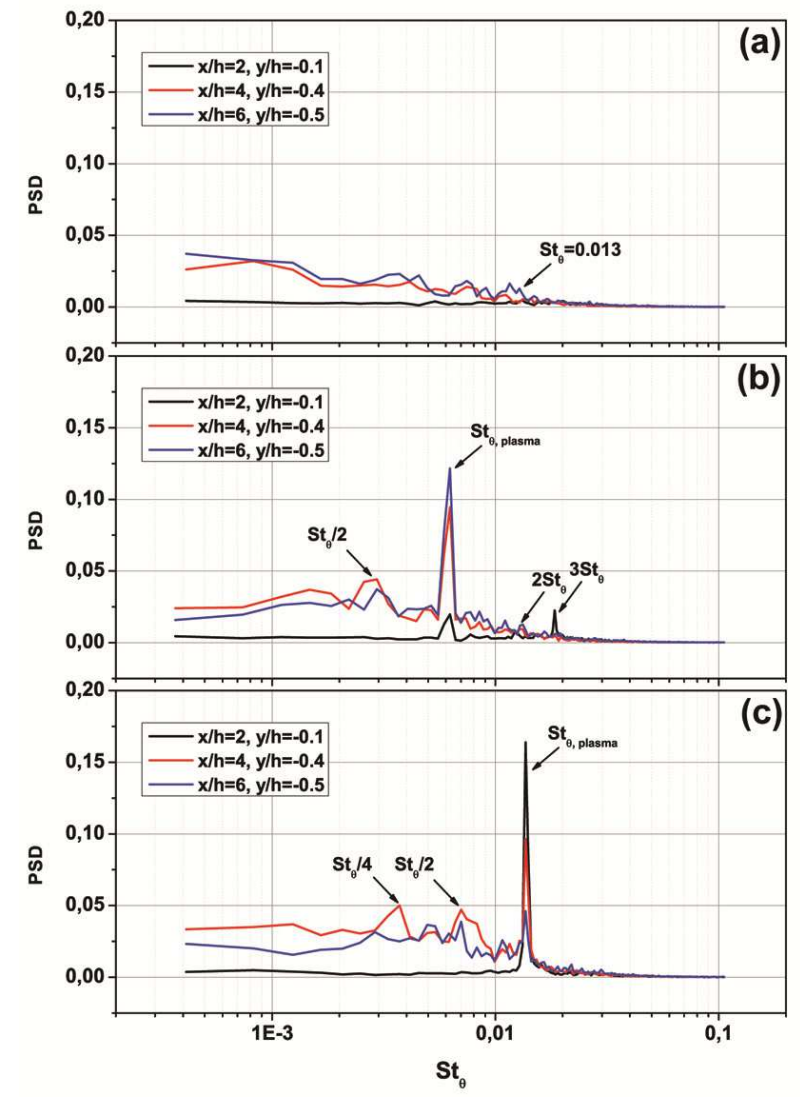

Figure 13 : Power density spectra of the fluctuating velocity component $v$ ' for the natural flow (a), plasma operated for excitation at $\mathrm{St}_{\theta}=\mathbf{0 . 0 0 6}$ (b) and plasma actuator imposing perturbations at $\mathrm{St}_{\theta}=\mathbf{0 . 0 1 3}$ (c). 

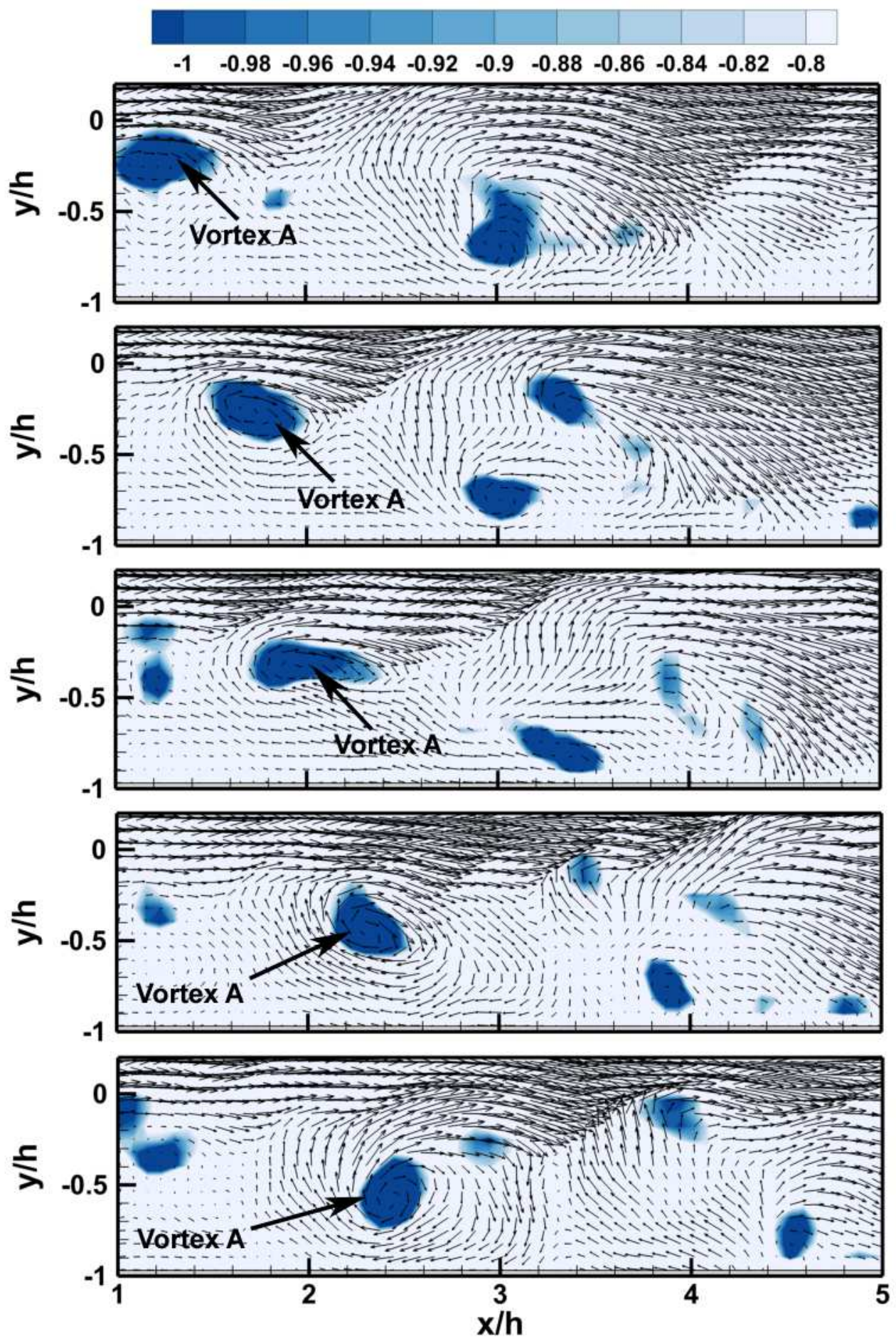

Figure 14 : Illustration of the vortex growing by rotational entrainment in case of actuation at $\mathrm{St}_{\theta}=0.006$. Each frame is separated by $2.5 \mathrm{~ms}$.

For the actuation at the shear layer mode $\left(\mathrm{St}_{\theta}=0.013\right)$, the organized development of the shear layer get new insight from the power density spectra analysis. In the earlier stage of the vortex formation $(\mathrm{x} / \mathrm{h}=2)$, the influence of the actuator is clearly evidenced by the high amplitude peak 
observed at the forcing frequency. The shear layer oscillations are fully periodic without a trace of high frequency content or low frequency vortex pairing. At $\mathrm{x} / \mathrm{h}=4$, the peak at the forcing frequency is largely damped. That suggests that the maximal amplification of the fundamental occurs upstream of $\mathrm{x} / \mathrm{h}=4$ (and upstream of the maximal amplification of the subharmonic, see the case of forcing at $\mathrm{St}_{\theta}=0.006$ introduced earlier in this paper) and that further downstream of this location the amplitude of the fundamental is decaying quickly. The PSDs also indicate that the vortex pairing process becomes significant from $\mathrm{x} / \mathrm{h}=4$ as demonstrated by the peaks at $\mathrm{St}_{\theta} / 2$ and $\mathrm{St}_{\theta} / 4$. The vortex pairing observed for shear layer mode forcing is illustrated in Figure 15. This sequence shows how two vortices (vortices $A$ and $B$ ) can pair at location $x / h \sim 3.5$. At the end of the amalgamation mechanism, the two structures have merged together to form a discrete coherent rotating region (vortex $\mathrm{A}+\mathrm{B})$. Finally, the pairing of vortices results in a single large-scale vortical flow structure that fills the space from the bottom wall up to the upper region of the separated shear layer. Due to the size of the flow structure and the entrainment of the convective flow, the residence time of large scale flow structures in the recirculating region is reduced. Both the reduced resident time and the promoted shedding process downstream of the mean reattachment point contribute to the periodic shrinkage of the recirculating region, this being partially responsible for the large reduction in the reattachment location. Furthermore, the amalgamation of adjacent vortices contributes to a larger spreading of the shear layer [47] as observed in Figure 11. The spreading mechanism, and the resulting reduced recirculation length, are also emphasized by the entrainment toward the recirculating region of nonturbulent flow initially close to the upper part of the shear layer but transported toward the bottom wall by the coherent flow structures. Furthermore, as it is indicated in [60], the pairing process also enhances the regions of flow trapped between two successive vortical flow structures increasing further the transfer from the non-turbulent region to the recirculation zone. Here, the time-resolved PIV measurements confirm that the large reduction in reattachment position derives from the increase in vortex pairing caused by the local forcing at the shear layer mode. 

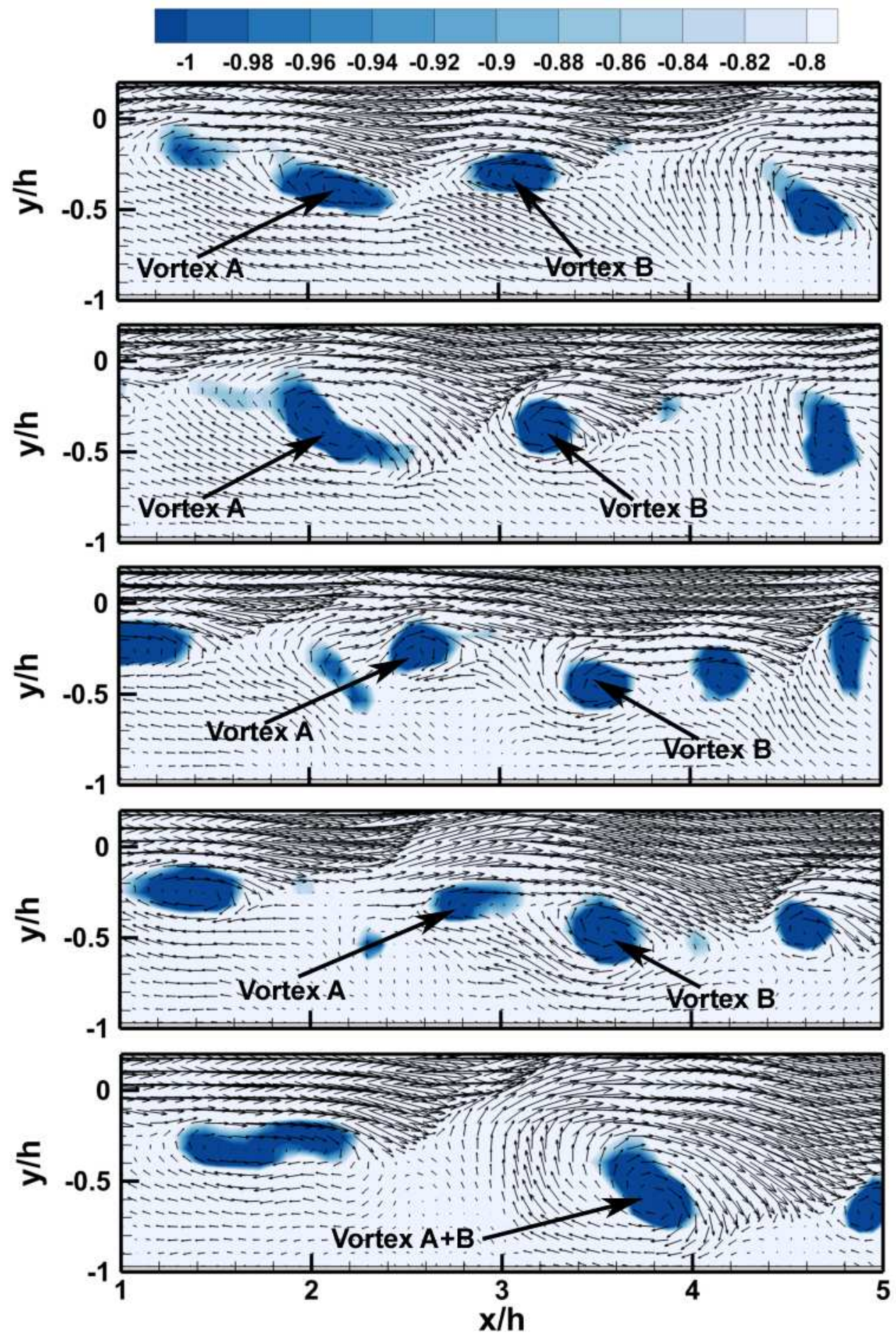

Figure 15 : Illustration of the vortex pairing mechanism in case of actuation at the shear layer mode $\left(\mathrm{St}_{\theta}=\mathbf{0 . 0 1 3}\right)$. Each frame is separated by $2.5 \mathrm{~ms}$.

\section{Concluding remarks}

The present experimental study interests in optimizing by an autonomous approach the effects of a linear plasma actuator on the vortex development of a separated shear layer formed in the wake of a 
backward-facing step. Here, the frequency, duty-cycle and amplitude of the imposed perturbations are simultaneously optimized regarding objective functions related to the mean flow reattachment location or to the level of the wall pressure fluctuations. The present study demonstrates that the reattachment length can be minimized or the wall fluctuating pressure maximized in only a few iterations, this with an electrical command autonomously optimized. However, the use of a GA code does not conduce to the definition of new control mechanisms as it can be expected by such an approach. Indeed, complimentary PIV measurements have shown that the two best forcing conditions identified by the GA approach correspond to the well-known excitations of the shear layer mode of instability of a turbulent shear layer (minimization of the reattachment) or to a periodic forcing at the first subharmonics of the most amplified mode (maximization of the wall fluctuating pressure coefficients). The time-resolved velocity fields indicate that periodic forcing by plasma discharge at the shear layer mode promotes a strong regularization of the vortex street. This forcing reduces largely the location of the mean reattachment point because of an enhanced vortex pairing mechanism. The present paper highlights the important role of the subharmonic instability for the development of the shear layer downstream of a BFS. Indeed, it was shown that large scale flow structures can be produced by forcing the flow at the first subharmonic of the shear layer mode. It has been shown that the amplification of the subharmonic continuously increases all along the shear layer development up to the reattachment point. This amplification corresponds to a vortex growing by both vortex pairing and fluid entrainment mechanisms as suggested by the time-resolved PIV measurements. These measurements have demonstrated that forcing by single-frequency at the subharmonic of the shear layer mode leads to the formation of flow structures with a vertical size corresponding to the height of the step mode; flow structures that are periodically shed in the wake of the BFS model and that persist beyond of the mean reattachment position.

Beyond simply using the robustness of an autonomous experimental optimization method, and its feasibility for wind tunnel experiments in context of flow control optimization, it is expected that autonomous seeking of optimal control conditions including multi-input and multi-objective capabilities would be of great interest for the flow control community. Indeed, in complex control 
scenario, for instance when several actuators are considered individually, when multi-frequency forcing with phase shift [61] or when the second objective function concerns a minimization of the consumed power as in [20], such algorithms may highlight unseen influence of some design variable on the flow and reveal new control mechanisms.

\section{Acknowledgment}

This work was supported by the $7^{\text {th }}$ Framework program FP7/2010-2013, MARS (grant agreement $\mathrm{n}^{\circ}$ 266326). Research equipment funded by the French Government program "Investissements d'Avenir" (LABEX INTERACTIFS, reference ANR-11-LABX-0017-01). The authors thank Ms P. Sujar-Garrido for his help in designing the step model and the fruitful discussions.

\section{References}

1- L.N. Cattafesta III and M. Sheplak, 'Actuators for active flow control', Annual Review of Fluid Mechanics 43, pp. 247-272, 2011

2- S. Obayashi, and S. Takanashi, 'Genetic optimization of target pressure distributions for inverse design methods, 'AIAA Journal 34, pp. 881-886, 1996

3- W. MacCormack, O.R. Tutty, E. Rogers, and P.A. Nelson, 'Stochastic optimisation based control of boundary layer transition', Control Engineering Practice 10, pp. 243-260, 2002

4- I. Rechenberg, 'Cybernetic Solution Path of an Experimental Problem'. Royal Aircraft Establishment, Library Translation 1122, Farnborough, 1965

5- N. Srinivas, and K. Deb, 'Multiobjective optimization using non dominated sorting in genetic algorithms,' Evolutionary Computation 2, pp. 221-248, 1994

6- X. Chen and R. K. Agarwal, 'Optimization of Wind Turbine Blade Airfoils Using a Multi-Objective Genetic Algorithm', Journal of Aircraft 50, pp. 519-527, 2013

7- M. Gad-El-Hak, 'Flow control: Passive, active, and reactive flow management,' Cambridge University press, 2007

8- D.E Goldberg, 'Genetic Algorithms in Search, Optimization, and Machine Learning,' Addison-Wesley, Boston, 1989.

9- R. Duvigneau, and M. Visonneau, 'Hybrid genetic algorithms and artificial neural networks for complex design optimization in CFD', Int. J. Numer. Meth. Fluids, 44, pp. 1257-1278, 2004.

10- J.F. Wang, J. Periaux, and M. Sefrioui, 'Parallel evolutionary algorithms for optimization problems in aerospace engineering,' J. Comput. Appl. Math., 149, pp.155-169, 2002.

11- A. Hilgers, and B.J. Boersma, 'Optimization of turbulent jet mixing,' Fluid Dynamics Research, 29, pp. 345-368, 2001

12- K. Morimoto, K. Iwamoto, Y. Suzuki, and N. Kasagi, 'Genetic algorithm-based optimization of feedback control scheme for wall turbulence,' Proc. 3rd Symp. Smart Control of Turbulence, pp 107-113, 2002

13- L. Huang, G. Huang, R. LeBeau and T. Hauser, 'Optimization of airfoil flow control using a genetic algorithm with diversity control,' J. Aircraft, 44, 2007

14- T.K. Sengupta, K. Deb, and S.B. Tala, 'Control of flow using genetic algorithm for a circular cylinder executing rotary oscillation,' Computers and Fluids, 36, pp.578-600, 2007. 
15- M. Milano, and P. Koumoutsakos, 'A clustering genetic algorithm for cylinder drag optimization,' J. Comp. Phys., Vol. 175, pp.79-107, 2002.

16- T. Watanabe, T. Tatsukawa, A.L. Jaimes, H. Aono, T. Nonomura, A. Oyama, and K. Fujii, 'Many-objective evolutionary computation for optimization of separated-flow control using a DBD plasma actuator,' Evolutionary Computation (CEC), 2014 IEEE Congress on, pp. 2849 - 2854, 2014.

17- J. Klockgether and H.P. Schwefel, 'Two-phase nozzle and hollow core jet experiments,' $11^{\text {th }}$ Symp. Engineering Aspects of Magnetohydrodynamics, Pasadena, 1970

18- M. Olhofer, D. Yankulova, and B. Sendhoff, 'Autonomous experimental design optimization of a flapping wing,' Genet. Program Evolvable Mach. 47, pp. 23-47, 2011

19- F. Boria, B. Stanford, W. Bowman, and Peter Ifju, 'Evolutionary optimization of a morphing wing with wind tunnel hardware-in-the-loop', AIAA paper 2009-1460

20- T. Sulaiman, S. Sekimoto, T. Tatsukawa, T. Nonomura, A. Oyama, and K. Fujii, 'DBD plasma actuator multiobjective design optimization at Reynolds number 63,000: Baseline case', Proc. ASME 2013, FEDSM2013-16325.

21- M. Kanazaki, T. Matsumo, K. Maeda, M. Kawazoe, 'Efficient global optimization applied to multi-objective design optimization of lift creating cylinder using plasma actuators', Proceedings of the 18th Asia Pacific Symposium on Intelligent and Evolutionary Systems, Volume 1, pp 663-677, 2015

22- V. Parezanovic, J.C. Laurentie, T. Duriez, C. Fourment, J. Delville, J.P. Bonnet, L. Cordier, B.R. Noack, M. Segond, M. Abel, T. Shaqarin, and S. Brunton, "Mixing layer manipulation experiment -- from periodic forcing to machine learning closed-loop control". Flow, Turbulence and Combustion 94, pp. 155-173, 2015

23- N Gautier, T Duriez, JL Aider, B Noack, M Segond, M Abel, 'Closed-loop separation control using machine learning,' J. of Fluid Mechanics 770, pp. 442- 457, 2015

24- T.C. Corke, C.L. enloe, and S.P. Wilkinson, 'Dielectric Barrier Discharge Plasma Actuators for Flow Control,' Annual Review of Fluid Mechanics 42, 2010.

25- Wang, J.-J., Choi, K.-S., Feng, L.-H. and Jukes, T.N., 'Recent developments in DBD plasma flow control,' Progress in Aerospace Sciences. 62, 52-78, 2013

26- N. Benard and E. Moreau, 'Electrical and mechanical characteristics of surface AC dielectric barrier discharge plasma actuators applied to airflow control,' Experiments in Fluids, 55, 2014

27- N. Benard, and E. Moreau, 'Capabilities of the dielectric barrier discharge plasma actuator for multi-frequency excitations', J. Phys. D: Appl. Phys. 43, 2010

28- N. Benard, P. Sujar-Garrido, E. Moreau, and J.P. Bonnet, 'Open loop control of a turbulent backward facing step by DBD,' Proc of TSFP 9, Melbourne, 2015

29- P. Sujar-Garrido, N. Benard, E. Moreau, J. P. Bonnet, 'Dielectric barrier discharge plasma actuator to control turbulent flow downstream of a backward-facing step,' Experiments in Fluids 56, 2015

30- D.M. Driver, H.L. Seegmiller and J.G. Marvin, 'Time-dependent behavior of reattaching shear layer', AIAA Journal 25, 1987

31- F.W. Roos, and J.T. Kegelman, 'Control of coherent structures in reattaching laminar and turbulent shear layers, ' AIAA Journal 24, 1986

32- S. Chun, Y.Z. Liu and H.J. Sung, 'Wall pressure fluctuations of a turbulent separated and reattaching flow affected by an unsteady wake,' Experiments in Fluids 37, pp.531-546, 2004

33- N.J. Cherry, R. Hillier, P. Latour, 'The unsteady structure of two-dimensional separated-and-reattaching flows,' $J$. Wind Eng. And Ind. Aerod. 11, pp. 95-105, 1983

34- Y.Z. Liu, W. Kang and H.J. Sung, 'Assessment of the organization of a turbulent separated and reattaching flow by measuring wall pressure fluctuations', Experiments in Fluids 38, pp. 485-493, 2005.

35- N.J. Cherry, R. Hillier, and P. Latour, 'Unsteady measurements in a separated and reattaching flow', Journal Fluid Mechanics 44, 1984. 
36- O. J. Selfridge, Pandemonium: 'A paradigm for learning', Proceedings of the Symposium on the Mechanization of Thought Processes, 511-529, 1959

37- W. W. Bledsoe, 'The use of biological concepts in the analytical study of systems', Paper presented at the ORSATIMS National Meeting, San Francisco, CA. 1961

38- D.S. Lee, C. Morillo, G. Bugeda, S. Oller, and E. Onate, 'Multilayered Composite Structure Design Optimisation using Distributed/Parallel Multi-Objective Evolutionary Algorithms', Journal of Composite Structures, 2011.

39- D.S. Lee, L.F. Gonzalez, J. Periaux, and G. Bugeda, 'Double Shock Control Bump Design Optimisation Using Hybridised Evolutionary Algorithms'. Special Issue Journal of Aerospace Engineering, Vol. 225, 2011.

40- J. d'Adamo, R. Sosa, and G. Artana, 'Active control of a backward facing step flow with plasma actuators,' Journal of Fluid Engineering, 136, 2014.

41- G. Correale, T. Michelis, and M. Kotsonis, 'NS-DBD plasma actuation on a backward facing step', AIAA paper 2014-0325.

42- K.B. Chun, and H.J. Sung, 'Control of turbulent separated flow over a backward-facing step by local forcing,' Experiments in Fluids, Vol. 21, pp. 417-426, 1996.

43- S. Bhattacharjee, B. Scheelke, and T.R. Troutt, 'Modification of vortex interactions in a reattaching separated flow,' AIAA J. 24, 1986.

44- Z. Mehrez, M. Bouterra, A. El Cafsi, A. Belghith, and P. Le Quere, 'Mass transfer control of a backward-facing step flow by local forcing-effect of reynolds number,' Thermal Science 15, pp. 367-378, 2011

45- M. Pastoor, L. Henning, B. R. Noack, R. King, and G. Tadmor. 'Feedback shear layer control for bluff body drag reduction,' J. Fluid Mech. 608, pp. 161-196, 2008.

46- M.A.Z Hasan, 'The flow over a backward-facing step under controlled perturbation: laminar separation,' J. Fluid Mechanics, 238, pp.73-96, 1992.

47- D. Oster, and I. Wygnanski, 'The forced mixing layer between parallel streams,' J. Fluid Mechanics 123, pp. 91130, 1982.

48- L.M. Hudy, A.M. Naguib, and W.M. Humphreys, 'Wall-pressure-array measurements beneath a separating/reattaching flow region', Physics of Fluids 15, 2003.

49- U.K. Kaul, 'First principle based PID control of mixing layer: Role of inflow perturbation spectrum,' AIAA paper 2014-2222.

50- N.N. Mansour, F. Hussain, and C. Buell, 'Subharmonic resonance in a mixing layer,' Center for Turbulent Research, Proc. of the summer program, 1988.

51- S. Kim, H. Choi, and J.Y. Yoo, 'Effect of local forcing on backward-facing step flow with laminar separation,' Journal of Turbulence 8, 2007.

52- C.O. Paschereit, and I. Wygnanski, 'Instabilities in the axisymmetric jet: subharmonic resonance,' in Advances in turbulence studies edited by Y. Unger and H. Branover (DOI 10.2514/4.866227), Progress in Astronautics and aeronautics, 1991

53- H.S. Husain, and F. Hussain, 'Experiments on subharmonic resonance in a shear layer', Journal of Fluid mechanics 304, pp. 343- 3721995

54- J. Cohen, and I. Wygnanski, 'The evolution of instabilities in the axisymmetric jet. Part 1. The linear growth of disturbances near the nozzle', Journal of Fluid Mechanics 176, pp. 191-219, 1987

55- I. Wygnanski, and R.A. Petersen, 'Coherent motion in excited free shear flows', AIAA Journal 25, pp. 201-213, 1987

56- C.M. Ho, and L.S. Huang, 'Subharmonics and vortex merging in mixing layers', Journal of Fluid Mechanics 119, 1982

57- H.Q. Zhang, and W. Shu, 'Numerical simulations of vortex merging and vortex splitting in mixing layer,' Science in China 33, 1990 
58- G.L. Brown, and A. Roshko, 'On density effects and large structure in turbulent mixing layers,' Journal of Fluid Mechanics 64, 1974.

59- L. Graftieaux, M. Michard, and N. Grosjean, 'Combining PIV, POD and vortex identification algorithms for the study of unsteady turbulent swirling flows', Measurements Sciences and Technology, Vol. 12, 1422, 2001

60- C.D. Winant, and F.K. Browand, 'Vortex pairing: the mechanism of turbulent layer growth at moderate Reynolds number,' J. Fluid Mechanics 63, pp. 237-255, 1974. 